\title{
Exponentials with infinite multiplicities
}

\author{
Alberto Carraro ${ }^{1,2}$, Thomas Ehrhard ${ }^{2, \star}$, and Antonino Salibra ${ }^{1, \star \star}$ \\ 1 Department of Computer Science, Ca' Foscari University, Venice \\ 2 PPS, UMR 7126, university Paris Diderot and CNRS
}

\begin{abstract}
Given a semi-ring with unit which satisfies some conditions, we define an exponential functor on the category of sets and relations which allows to define a denotational model of Differential Linear Logic and of the lambda-calculus with resources. We show that, when the semiring has an element which is infinite in the sense that it is equal to its successor, this model does not validate the Taylor formula and that it is possible to build, in the associated Kleisli cartesian closed category, a model of the pure lambda-calculus which is not sensible. This is a quantitative analogue of the standard graph model construction in the category of Scott domains.

Keywords: lambda-calculus, linear logic, denotational semantics, differential lambda-calculus, resource lambda-calculus, non sensible models.
\end{abstract}

\section{Introduction}

The category of sets and relations is a quite standard denotational model of Linear Logic which underlies most denotational models of this system (coherence spaces, hypercoherence spaces, totality spaces, finiteness spaces...). In this completely elementary setting, a formula is interpreted as a set, and a proof of that formula is interpreted as a subset of the set interpreting the formula.

Logical connectives are interpreted very simply: tensor product, par and linear implication are interpreted as cartesian products whereas direct product (with) and direct sums (plus) are interpreted as disjoint union. The linear negation of a set is the same set: it is a remarkable feature of Linear Logic that it admits such a "degenerate" semantics of types, which is nonetheless non trivial in the sense that proofs are not identified.

Exponentials are traditionally interpreted by the operation which maps a set $X$ to the set of all finite multisets of elements of $X$. One might be tempted to use finite sets instead of finite multisets since, in the coherence space semantics, the exponential can be interpreted by an operation which maps a coherence space to the sets of its finite cliques (with a suitable coherence). In the relational model however, such an interpretation of the exponentials based on finite sets is not possible as it leads to a dereliction which is not natural (in the categorical sense).

With this standard multiset-based interpretation of exponentials, the relational model interprets also the differential extensions of Linear Logic and of

\footnotetext{
* Work partially funded by the ANR project CHOCO.

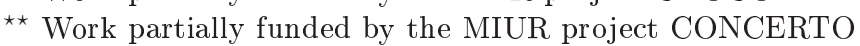


the lambda-calculus presented in [ER03,ER04,EL09]. In this extension of the lambda-calculus, terms can be derived (differentiated): a term $M$ of type $A \rightarrow B$ can be transformed into a term $M^{\prime}$ of type $A \rightarrow(A \rightarrow B)$ which is linear in its second parameter of type $A$. The word "linear" can be taken here in its standard algebraic sense, or in its operational sense of "using its argument exactly once". This differentiation operation can be iterated, yielding a $n$th derivative $M^{(n)}: A \rightarrow\left(A^{n} \rightarrow B\right)$ which is $n$-linear in its $n$ last arguments of type $A$. The introduction of this new construction requires the possibility of freely adding terms of the same type: in the model Rel, this addition operation is interpreted as set union (remember that terms as interpreted as subsets of the interpretations of types). Also, each type has to contain a 0 element which, here, is the empty set.

This strongly suggests to consider the following "Taylor series", given a term $M$ of type $A \rightarrow B$ and a term $N$ of type $A: \sum_{n=0}^{\infty} \frac{1}{n !} M^{(n)}(0) \cdot(N, \ldots, N)$. For simplifying the setting and for dealing easily with untyped terms, it is suitable to consider a version of that formula where coefficients are all equal to one, and where addition of terms is an idempotent operation: terms form a complete lattice and the Taylor expansion of $M$ can be written more simply $\bigvee_{n=0}^{\infty} M^{(n)}(0)$. $(N, \ldots, N)$.

With Regnier, the second author studied this operation in [ER08,ER06a], introducing a lambda-calculus with resources which can be seen as the differential lambda-calculus where ordinary ${ }^{3}$ application can be used only for applying a term to 0: this is the only ordinary application needed if we want to Taylor expand all the applications occurring in lambda-terms. In these two papers we proved in an untyped setting that, Taylor expanding completely a lambda-term $M$, one obtains a (generally infinite) linear combination of resource terms and that, if one normalizes each resource term occurring in that formal sum ${ }^{4}$, one obtains the Taylor expansion of the Böhm tree of $M$.

This result implies that, in a denotational model which validates the Taylor expansion formula in the sense that the interpretation of a term $M$ is equal to the interpretation of its Taylor expansion, the interpretation of an unsolvable lambda-term ${ }^{5}$ is necessarily equal to 0 . Since the multiset-based exponential of Rel validates the Taylor expansion formula, any model of the pure lambdacalculus in the corresponding cartesian closed category, such as the model presented in [BEM07,BEM09], seems to be bound to be sensible (at least if differential operations are interpreted in the standard way). This seems to be a serious limitation in the equational expressive power of this kind of semantics.

\footnotetext{
${ }^{3}$ In the differential lambda-calculus, there are two kinds of application: the ordinary application of a term to an argument, and the application of the $n$th derivative of a term to a $n$-tuple of terms. This latter application is $n$-linear in its arguments whereas the former is not linear.

${ }^{4}$ Resource terms are strongly normalizing, even if they are not typeable.

${ }^{5}$ We recall that a term is solvable iff its head reduction terminates.
} 
This problem arised during a general investigation undertaken by the authors, whose scope is to develop an algebraic setting for differential extensions of the lambda-calculus, in the spirit of [PS98,MS09].

Content. The present paper proposes a solution to this problem, by introducing new exponential operations on Rel. The idea is quite simple: we replace the set $\mathbb{N}$ of natural numbers (which are used for counting multiplicities of elements in multisets) by more general semi-rings which typically contain "infinite elements" $\omega$ such that $\omega+1=\omega$. Mutatis mutandis, the various structures of the exponentials (functorial action, dereliction etc) are interpreted as with the ordinary multiset-based exponentials. For these structures to satisfy the required equations, some rather restrictive conditions have to be satisfied by the considered semi-ring: the semi-rings which satisfy these conditions are called "multiplicity semi-rings". We show that such a semi-ring must contain $\mathbb{N}$ and we exhibit multiplicity semi-rings with infinite elements.

In these models with infinite multiplicities, the differential constructions are available, but the Taylor formula does not hold. It is possible to find morphisms $f: A \rightarrow B$ (in the associated cartesian closed category) which are $\neq 0$ but are such that, for all $n$, the $n$th derivative $f^{(n)}(0): A^{n} \rightarrow B$ is equal to 0 . The Taylor expansion of such a function is the 0 map, and hence the function is different from its Taylor expansion. This is analogous to the well known smooth $\left(C^{\infty}\right)$ map $f: \mathbb{R} \rightarrow \mathbb{R}$ defined by $f(0)=0$ and $f(x)=e^{-1 /|x|}$ for $x \neq 0$ : all the derivatives of $f$ at 0 are equal to 0 and hence there is no neighborhood of 0 where $f$ coincides with its Taylor expansion at 0 . In some sense, $f$ is infinitely flat at 0 , and we obtain a similar effect with our infinite multiplicities.

For any multiplicity semi-ring which contains an infinite element, we build a model of the pure lambda-calculus, which is not sensible and, more precisely, where the term $\Omega=(\lambda x(x) x) \lambda x(x) x$ has a non-empty interpretation (we also exhibit a non solvable term whose interpretation is distinct from that of $\Omega$ ).

Warning. Most omitted proofs can be found in the Appendix section.

\section{The relational model of Linear Logic}

Rel is the category whose objects are sets and with hom-sets $\operatorname{Rel}(X, Y)=$ $\mathcal{P}(X \times Y)$. In this category, composition is the ordinary composition of relations: if $R \in \operatorname{Rel}(X, Y)$ and $S \in \operatorname{Rel}(Y, Z)$, then

$$
S \cdot R=\{(a, c) \in X \times Z \mid \exists b \in Y(a, b) \in R \text { and }(b, c) \in S\} .
$$

and identities are the diagonal relations: $\operatorname{ld}_{X}=\{(a, a) \in X\}$.

This category has a well known symmetric monoidal structure (compact closed actually), with tensor product given on objects by $X_{1} \otimes X_{2}=X_{1} \times X_{2}$ and on morphisms by

$$
R_{1} \otimes R_{2}=\left\{\left(\left(a_{1}, a_{2}\right),\left(b_{1}, b_{2}\right)\right) \mid\left(a_{i}, b_{i}\right) \in R_{i} \text { for } i=1,2\right\}
$$


for any $R_{i} \in \operatorname{Rel}\left(X_{i}, Y_{i}\right)(i=1,2)$. The associativity and symmetry isomorphisms are the obvious bijections, the neutral object of the tensor product is the singleton set $1=\{*\}$.

This monoidal category is closed: the object of morphisms from $X$ to $Y$ is $X \multimap Y=X \times Y$, with evaluation morphism ev $\in \operatorname{Rel}((X \multimap Y) \otimes X, Y)$ given by ev $=\{(((a, b), a), b) \mid a \in X, b \in Y\}$. And, given $R \in \operatorname{Rel}(Z \otimes X, Y)$, the linear curryfication of $R$ is $\operatorname{cur}(R)=\operatorname{Rel}(Z, X \multimap Y)$. This category is starautonomous, with dualizing object $\perp=1$.

The category Rel is also cartesian: the cartesian product of a family of objects $\left(X_{i}\right)_{i \in I}$ is $\prod_{i \in I} X_{i}=\bigcup_{i \in I}(\{i\} \times I)$. The binary cartesian product of $X$ and $Y$ is denoted as $X \& Y$ and the terminal object is $\top=\emptyset$. The projection $\pi_{i} \in \operatorname{Rel}\left(\prod_{i \in I} X_{i}, X_{i}\right)$ is $\pi_{i}=\left\{((i, a), a) \mid a \in X_{i}\right\}$ and, given a family $\left(R_{i}\right)_{i \in I}$ of morphisms $R_{i} \in \operatorname{Rel}\left(Y, X_{i}\right)$, the corresponding morphism $\left\langle R_{i}\right\rangle_{i \in I} \in$ $\operatorname{Rel}\left(Y, \prod_{i \in I} X_{i}\right)$ is given by $\left\langle R_{i}\right\rangle_{i \in I}=\left\{(b,(i, a)) \mid i \in I\right.$ and $\left.(b, a) \in R_{i}\right\}$.

\section{Exponentials}

We present a way of building exponential functors, once a notion of multiplicity is given, as a semi-ring satisfying strong conditions.

\subsection{Multiplicity semi-rings}

Notational convention for indices. We shall use quite often multiple indices, written as subscript as in " $a_{i j k}$ " which has three indices $i, j$ and $k$. When there are no ambiguities, these indices will not be separated by commas. We insert commas when we use multiplication on these indices, as in " $a_{i, 2 j, k}$ " for instance.

A semi-ring $M$ is a multiplicity semi-ring if it is commutative, has a multiplicative unit and satisfies

(MS1) $\forall n_{1}, n_{2} \in M \quad n_{1}+n_{2}=0 \Rightarrow n_{1}=n_{2}=0$ (we say that $M$ is positive)

(MS2) $\forall n_{1}, n_{2} \in M \quad n_{1}+n_{2}=1 \Rightarrow n_{1}=0$ or $n_{2}=0$ (we say that $M$ is discrete)

(MS3) $\forall n_{1}, n_{2}, p_{1}, p_{2} \in M \quad n_{1}+n_{2}=p_{1}+p_{2} \Rightarrow \exists r_{11}, r_{12}, r_{21}, r_{22} \in M \quad n_{1}=$ $r_{11}+r_{12}, n_{2}=r_{21}+r_{22}, p_{1}=r_{11}+r_{21}, p_{2}=r_{12}+r_{22}$ (we say that $M$ has the additive splitting property)

(MS4) $\forall m, p, n_{1}, n_{2} \in M \quad p m=n_{1}+n_{2} \Rightarrow \exists p_{1}, p_{2}, m_{11}, m_{12}, m_{21}, m_{22} \in M \quad m_{11}+$ $m_{21}=m_{12}+m_{22}=m, p_{1} m_{11}+p_{2} m_{12}=n_{1}, p_{1} m_{21}+p_{2} m_{22}=n_{2}$ and $p_{1}+$ $p_{2}=p$ (we say that $M$ has the multiplicative splitting property).

Generalized splitting properties. The splitting conditions are expressed in a binary way, we must generalize them to arbitrary arities. We first generalize Condition (MS3).

Lemma 1. Let $M$ be a semi-ring which has the splitting property. Let $n_{1}, \ldots, n_{l} \in$ $M$ and $p_{1}, \ldots, p_{r} \in M$ be such that $\sum_{i=1}^{l} n_{i}=\sum_{j=1}^{r} p_{j}$. Then there is a family $\left(s_{i j}\right)_{i=1, j=1}^{l, r}$ of elements of $M$ such that $\forall i \in\{1, \ldots, l\} n_{i}=\sum_{j=1}^{r} s_{i j}$ and $\forall j \in\{1, \ldots, r\} p_{j}=\sum_{i=1}^{l} s_{i j}$. 
Similarly, we generalize Condition (MS4).

Lemma 2. Let $M$ be a semi-ring which has the multiplicative and the additive splitting properties. Let $k \in \mathbb{N}$ with $k \neq 0$. Let $l=2^{k-1}$. For all $n_{1}, \ldots, n_{k}, m, p \in$ $M$, if $n_{1}+\cdots+n_{k}=m p$, then there exist $\left(p_{j}\right)_{j=1}^{l} \in M$ and $\left(m_{i j}\right)_{i=1, j=1}^{k, l}$ with

- $p_{1}+\cdots+p_{l}=p$

- $m_{1 j}+\cdots+m_{k j}=m$ for $j=1, \ldots, l$

- and $m_{i 1} p_{1}+\cdots+m_{i l} p_{l}=n_{i}$ for $i=1, \ldots, k$.

In particular, a multiplicity semi-ring cannot be finite.

Proposition 1. Any multiplicity semi-ring $M$ contains an isomorphic copy of $\mathbb{N}$.

We shall simply say that $M$ contains $\mathbb{N}$, that is $\mathbb{N} \subseteq M$. In particular, a multiplicity semi-ring cannot be finite.

Examples of multiplicity semi-rings. The elements of a multiplicity semiring should be considered as generalized natural numbers. We give here examples of semi-ring satisfying these axioms.

Natural numbers. The most canonical example of multiplicity semi-ring is the set $\mathbb{N}$ of natural numbers, with the ordinary addition and multiplication.

Proposition 2. $\mathbb{N}$ is a multiplicity semi-ring.

Completed natural numbers. Let $\overline{\mathbb{N}}=\mathbb{N} \cup\{\omega\}$ be the "completed set of natural numbers". We extend addition to this set by $n+\omega=\omega+n=\omega$, and multiplication by $0 \omega=\omega 0=0$ and $n \omega=\omega n=\omega$ for $n \neq 0$.

Proposition 3. $\overline{\mathbb{N}}$ is a multiplicity semi-ring.

A semi-ring with infinite and non-idempotent elements. A more interesting example is $\mathbb{N}_{2}=\left(\mathbb{N}^{+} \times \mathbb{N}\right) \cup\{0\}$. The element $(n, d)$ of this set (with $\left.n \neq 0\right)$ will be denoted as $n \omega^{d}$. We extend this notation to the case where $n=0$, identifying $0 \omega^{d}$ with 0 , which is quite natural with these notations. Addition is defined as follows (0 being of course neutral for this operation)

$$
n \omega^{d}+n^{\prime} \omega^{d^{\prime}}= \begin{cases}\left(n+n^{\prime}\right) \omega^{d} & \text { if } d=d^{\prime} \\ n \omega^{d} & \text { if } n \neq 0 \text { and } d^{\prime}<d \\ n^{\prime} \omega^{d^{\prime}} & \text { if } n^{\prime} \neq 0 \text { and } d<d^{\prime}\end{cases}
$$

and multiplication is defined by $n \omega^{d} n^{\prime} \omega^{d^{\prime}}=n n^{\prime} \omega^{d+d^{\prime}}$.

Proposition 4. $\mathbb{N}_{2}$ is a multiplicity semi-ring.

From now on, $\mathbb{M}$ denotes a multiplicity semi-ring. 


\subsection{The exponential functor}

Given a set $X$, we define $!_{\mathbb{M}} X$ as the free $\mathbb{M}$-module $\mathbb{M}\langle X\rangle$ generated by $X$, that is, as the set of all functions $\mu: X \rightarrow \mathbb{M}$ such that $\operatorname{supp}(\mu)=\{a \in X \mid \mu(a) \neq$ $0\}$ (the support of $\mu$ ) is finite. These functions will be called $\mathbb{M}$-multisets (of elements of $X$ ).

Given $a \in X$, we denote as $[a] \in !_{\mathbb{M}} X$ the function given by $[a](b)=\delta_{a, b}$. We use the standard algebraic notations for denoting the operations in the $\mathbb{M}$-module $!_{\mathbb{M}} X$. If $\mu \in !_{\mathbb{M}} X$, we define the cardinality of $\mu$ by $\# \mu=\sum_{a \in \operatorname{supp}(\mu)} \mu(a) \in \mathbb{M}$.

Given $R \in \operatorname{Rel}(X, Y)$, we define $!_{\mathbb{M}} R \in \operatorname{Rel}\left(!_{\mathbb{M}} X, !_{\mathbb{M}} Y\right)$ as the set of all pairs $(\mu, \nu)$ such that one can find $\sigma \in \mathbb{M}\langle X \times Y\rangle$ with $\operatorname{supp}(\sigma) \subseteq R$ and

$$
\forall a \in X \quad \mu(a)=\sum_{b \in Y} \sigma(a, b) \quad \text { and } \quad \nu(b)=\sum_{a \in X} \sigma(a, b) .
$$

We say then that $\sigma$ is a witness of $(\mu, \nu)$ for $R$. Observe that all these sums are finite because $\sigma \in \mathbb{M}\langle X \times Y\rangle$.

It is clear from this definition that $!_{\mathbb{M}} I d=I d$.

Lemma 3. Let $R \in \operatorname{Rel}(X, Y)$ and $S \in \operatorname{Rel}(Y, Z)$. Then $!_{\mathbb{M}}(S \cdot R)=!_{\mathbb{M}} S \cdot !_{\mathbb{M}} R$.

Proof. This is essentially an application of Lemma 1, see the Appendix.

Lemma 4. Let $R \subseteq X \times Y$ and let $\left(\mu_{i}, \nu_{i}\right) \in !_{\mathbb{M}} R$ and $q_{i} \in \mathbb{M}$ for $i=1, \ldots, n$. Then $\left(\sum_{i=1}^{n} p_{i} \mu_{i}, \sum_{i=1}^{n} p_{i} \nu_{i}\right) \in !_{\mathbb{M}} R$.

Proof. For each $i$, choose a witness $\sigma_{i}$ of $\left(\mu_{i}, \nu_{i}\right)$ for $R$. Then $\sum_{i=1}^{n} p_{i} \sigma_{i}$ is a witness of $\left(\sum_{i=1}^{n} p_{i} \mu_{i}, \sum_{i=1}^{n} p_{i} \nu_{i}\right)$ for $R$.

\subsection{Comonad structure of the exponential}

We introduce the fundamental comonadic structure of the exponential functor, which consists of two natural transformations usually called dereliction (the counit of the comonad) and digging (the comultiplication of the comonad).

Dereliction. We set $\mathrm{d}_{X}=\{([a], a) \mid a \in X\} \in \operatorname{Rel}\left(!_{\mathbb{M}} X, X\right)$.

Lemma 5. $\mathrm{d}_{X}$ is a natural transformation from $!_{\mathbb{M}}$ to $\mathrm{ld}$.

Proof. One applies Conditions (MS1) and (MS2), see the Appendix.

Remark 1. One could consider taking $\mathbb{M}=\{0,1\}$ with $1+1=1$, and then we would have $!_{\mathbb{M}} X=\mathcal{P}_{\text {fin }}(X)$, the set of all finite subsets of $X$. But this semiring does not satisfy Condition (MS2) and, indeed, dereliction is not natural as already mentioned in the introduction. 
Digging. This operation is more problematic and some preliminaries are required.

Lemma 6. Let $X$ and $Y$ be sets and let $R \subseteq X \times Y$. Let $\nu_{1}, \nu_{2} \in !_{\mathbb{M}} Y$ and $\mu \in !_{\mathbb{M}} X$. If $\left(\mu, \nu_{1}+\nu_{2}\right) \in !_{\mathbb{M}} R$, then one can find $\mu_{1}, \mu_{2} \in !_{\mathbb{M}} X$ such that $\mu_{1}+\mu_{2}=\mu$ and $\left(\mu_{i}, \nu_{i}\right) \in !_{\mathbb{M}} R$ for $i=1,2$.

Proof. We use Lemma 1, see the Appendix.

Given $M \in !_{\mathbb{M} !} !_{\mathbb{M}} X$, we set

$$
\Sigma(M)=\sum_{m \in !_{M} X} M(m) m .
$$

Since $M$ has a finite support, this sum is actually a finite sum (the linear combination, with coefficients $M(m) \in \mathbb{M}$, is taken in the module $\left.!_{\mathbb{M}} X\right)$.

We define $\mathrm{p}_{X} \in \operatorname{Rel}\left(!_{\mathbb{M}} X, !_{\mathbb{M}} !_{\mathbb{M}} X\right)$ by

$$
\mathrm{p}_{X}=\left\{(\Sigma(M), M) \mid M \in !_{\mathbb{M}} !_{\mathbb{M}} X\right\} .
$$

The next lemma is the main tool for proving the naturality of digging. It combines the two generalized splitting properties of $\mathbb{M}$.

Lemma 7. Let $X$ and $Y$ be sets and let $R \subseteq X \times Y$ be finite. There exists $q(R) \in \mathbb{N}$ with the following property: for any $\mu \in !_{\mathbb{M}} X, \pi \in !_{\mathbb{M}} Y$ and $p \in \mathbb{M}$, if $(\mu, p \pi) \in !_{\mathbb{M}} R$, then one can find $p_{1}, \ldots, p_{q(R)} \in \mathbb{M}$ and $\mu_{1}, \ldots, \mu_{q(R)} \in !_{\mathbb{M}} X$ such that $\sum_{j=1}^{q(R)} p_{j}=p, \sum_{j=1}^{q(R)} p_{j} \mu_{j}=\mu$ and $\left(\mu_{j}, \pi\right) \in !_{\mathbb{M}} R$ for each $j=1, \ldots, q(R)$.

Proof. Let $I=\{a \in X \mid \exists b \in Y(a, b) \in R\}$ and $J=\{b \in Y \mid \exists a \in X(a, b) \in R\}$. Given $b \in J$, let $\operatorname{deg}_{b}(R)=\#\{a \in X \mid(a, b) \in R\}-1 \in \mathbb{N}$ and let $\operatorname{deg}(R)=$ $\sum_{b \in J} \operatorname{deg}_{b}(R)$. We prove the result by induction on $\operatorname{deg}(R)$.

Assume first that $\operatorname{deg}(R)=0$, so that, for any $b \in J$, there is exactly one $a \in I$ such that $(a, b) \in R$, let us set $a=g(b): g$ is a surjective function from $J$ to $I$ whose graph coincides with $R$ (in the sense that $R=\{(g(b), b) \mid b \in J\}$ ). Let $\sigma$ be a witness of $(\mu, p \pi)$ for $R$. For all $b \in J$ we have $p \pi(b)=\sum_{a \in X} \sigma(a, b)=$ $\sigma(g(b), b)$ and for all $a \in I$ we have $\mu(a)=\sum_{g(b)=a} \sigma(a, b)=p \sum_{g(b)=a} \pi(b)$. Let $\tau \in \mathbb{M}\langle X \times Y\rangle$ be defined by

$$
\tau(a, b)= \begin{cases}\pi(b) & \text { if } g(b)=a \\ 0 & \text { otherwise }\end{cases}
$$

then clearly $\operatorname{supp}(\tau) \subseteq R$ and $\tau$ is a witness of $\left(\mu^{\prime}, \pi\right)$ for $R$, where $\mu^{\prime} \in !_{\mathbb{M}} X$ is given by $\mu^{\prime}(a)=\sum_{g(b)=a} \pi(a)$. Since $p \mu^{\prime}=\mu$, we obtained the required property (with $q(R)=1, p_{1}=p$ and $\mu_{1}=\mu^{\prime}$ ).

Assume now that $\operatorname{deg}(R)>0$ and let us pick some $b \in J$ such that $k=$ $\operatorname{deg}_{b}(R)+1>1$. Let $a_{1}, \ldots, a_{k}$ be a repetition-free enumeration of the elements $a$ of $I$ such that $(a, b) \in R$. We have

$$
p \pi(b)=\sum_{i=1}^{k} \sigma\left(a_{i}, b\right) .
$$


Let $l=2^{k}$. By Lemma 2, there exist $\left(p_{j}\right)_{j=1}^{l} \in \mathbb{M}^{l}$ and $\left(m_{i j}\right)_{i=1, j=1}^{k, l}$ elements of $M$ with

- $p_{1}+\cdots+p_{l}=p$

- $m_{1 j}+\cdots+m_{k j}=\pi(b)$ for $j=1, \ldots, l$

- and $m_{i 1} p_{1}+\cdots+m_{i l} p_{l}=\sigma\left(a_{i}, b\right)$ for $i=1, \ldots, k$.

Let $b_{1}, \ldots, b_{k}$ be pairwise distinct new elements, which do not belong to $X$ nor to $Y$, and let $Y^{\prime}=(Y \backslash\{b\}) \cup\left\{b_{1}, \ldots, b_{k}\right\}$. We define a new relation to which we'll be able to apply the inductive hypothesis as follows:

$$
S=\left\{\left(a, b^{\prime}\right) \in R \mid b^{\prime} \neq b\right\} \cup\left\{\left(a_{i}, b_{i}\right) \mid i=1, \ldots, k\right\} .
$$

Then we have $\operatorname{deg}(S)=\operatorname{deg}(R)-k+1<\operatorname{deg}(R)$. Let $\tau \in \mathbb{M}\left\langle X \times Y^{\prime}\right\rangle$ be given by

$$
\tau(a, c)= \begin{cases}\sigma(a, c) & \text { if } c \notin\left\{b_{1}, \ldots, b_{k}\right\} \\ \sigma\left(a_{i}, b\right) & \text { if } c=b_{i} \text { and } a=a_{i} \\ 0 & \text { otherwise. }\end{cases}
$$

It is clear that $\operatorname{supp}(\tau) \subseteq S$. Moreover, $\tau$ is a witness of $\left(\mu, \sum_{j=1}^{l} p_{j} \pi_{j}\right)$ for $S$, where $\pi_{j} \in !_{\mathbb{M}} Y^{\prime}$ is given by

$$
\pi_{j}(c)= \begin{cases}\pi(c) & \text { if } c \notin\left\{b_{1}, \ldots, b_{k}\right\} \\ m_{i j} & \text { if } c=b_{i} .\end{cases}
$$

for each $j \in\{1, \ldots, l\}$. Indeed, for $a \in X$ we have

$$
\begin{aligned}
\sum_{c \in Y^{\prime}} \tau(a, c) & =\sum_{c \in Y^{\prime} \backslash\left\{b_{1}, \ldots, b_{k}\right\}} \tau(a, c)+\sum_{i=1}^{k} \tau\left(a, b_{i}\right) \\
& =\sum_{c \in Y^{\prime} \backslash\left\{b_{1}, \ldots, b_{k}\right\}} \sigma(a, c)+\sum_{i=1}^{k} \delta_{a, a_{i}} \sigma\left(a_{i}, b\right) \\
& =\sum_{c \in Y^{\prime} \backslash\left\{c_{1}, \ldots, c_{k}\right\}} \sigma(a, c)+\sigma(a, b)=\sum_{b \in Y} \sigma(a, b)=\mu(a)
\end{aligned}
$$

and for $c \in Y^{\prime} \backslash\left\{b_{1}, \ldots, b_{k}\right\}$ we have

$$
\sum_{a \in X} \tau(a, c)=\sum_{a \in X} \sigma(a, c)=p \pi(c)=\sum_{j=1}^{l} p_{j} \pi_{j}(c) \quad \text { since }\left\{\begin{array}{l}
\forall j \pi_{j}(c)=\pi(c) \\
\sum_{j=1}^{l} p_{j}=p
\end{array}\right.
$$

and last, for $c=b_{i}$ (with $i \in\{1, \ldots, k\}$ ), we have

$$
\sum_{a \in X} \tau(a, c)=\sigma\left(a_{i}, b\right)=\sum_{j=1}^{l} m_{i j} p_{j}=\sum_{j=1}^{l} p_{j} \pi_{j}(c) .
$$


By Lemma 6 , since $\left(\mu, \sum_{j=1}^{l} p_{j} \pi_{j}\right) \in !_{\mathbb{M}} S$, we can find $\mu_{1}, \ldots, \mu_{l} \in !_{\mathbb{M}} X$ such that $\sum_{j=1}^{l} \mu_{j}=\mu$ and $\left(\mu_{j}, p_{j} \pi_{j}\right) \in !_{\mathbb{M}} S$ for each $j \in \bar{l}$. Since $\operatorname{deg}(S)<\operatorname{deg}(R)$, we can apply the inductive hypothesis for each $j \in \bar{l}$. So we can find a family $\left(p_{j s}\right)_{j=1, s=1}^{l, q(S)}$ of elements of $\mathbb{M}$ such that $p_{j}=\sum_{s=1}^{q(S)} p_{j s}$ and we can find a family $\left(\mu_{j s}\right)_{j=1, h=1}^{l, q(S)}$ of elements of $!_{\mathbb{M}} X$ such that $\sum_{s=1}^{q(S)} p_{j s} \mu_{j s}=\mu_{j}$, and moreover $\left(\mu_{j s}, \pi_{j}\right) \in !_{\mathbb{M}} S$ for each $j \in \bar{l}$ and $s \in \overline{q(S)}$. We conclude the proof by showing that $\left(\mu_{j s}, \pi\right) \in !_{\mathbb{M}} R$. Let $\tau_{j s} \in \mathbb{M}\left\langle X \times Y^{\prime}\right\rangle$ be a witness of $\left(\mu_{j s}, \pi_{j}\right)$ for $S$. Let $\sigma_{j s} \in \mathbb{M}\langle X \times Y\rangle$ be given by

$$
\sigma_{j s}\left(a, b^{\prime}\right)= \begin{cases}\tau_{j s}\left(a, b^{\prime}\right) & \text { if } b^{\prime} \neq b \\ \sum_{i=1}^{k} \tau_{j s}\left(a, b_{i}\right) & \text { if } b^{\prime}=b\end{cases}
$$

For $b^{\prime} \in Y \backslash\{b\}$, we have $\sum_{a \in X} \sigma_{j s}\left(a, b^{\prime}\right)=\sum_{a \in X} \tau_{j s}\left(a, b^{\prime}\right)=\pi_{j}\left(b^{\prime}\right)=\pi\left(b^{\prime}\right)$. Next we have

$$
\begin{aligned}
\sum_{a \in X} \sigma_{j s}(a, b) & =\sum_{a \in X} \sum_{i=1}^{k} \tau_{j s}\left(a, b_{i}\right) \\
& =\sum_{i=1}^{k} \sum_{a \in X} \tau_{j s}\left(a, b_{i}\right) \\
& =\sum_{i=1}^{k} \pi_{j}\left(b_{i}\right)=\sum_{i=1}^{k} m_{i j}=\pi(b) .
\end{aligned}
$$

On the other hand we have

$$
\begin{aligned}
\sum_{b^{\prime} \in Y} \sigma_{j s}\left(a, b^{\prime}\right) & =\sum_{b^{\prime} \in Y \backslash\{b\}} \sigma_{j s}\left(a, b^{\prime}\right)+\sigma_{j s}(a, b) \\
& =\sum_{b^{\prime} \in Y \backslash\{b\}} \tau_{j s}\left(a, b^{\prime}\right)+\sum_{i=1}^{k} \tau_{j s}\left(a, b_{i}\right) \\
& =\sum_{c \in Y^{\prime}} \tau_{j s}(a, c)=\mu_{j s}(a) .
\end{aligned}
$$

It remains to prove that $\operatorname{supp}\left(\sigma_{j s}\right) \subseteq R$, but this results immediately from the definition of $\sigma_{j s}$ and from the fact that $\operatorname{supp}\left(\tau_{j s}\right) \subseteq S$.

Observe that we can take $q(R)=l q(S)$, so that in general $q(R)=2^{\operatorname{deg}(R)}$.

Lemma 8. $\mathrm{p}_{X}$ is a natural transformation from $!_{\mathbb{M}}$ to $!_{\mathbb{M}} !_{\mathbb{M}}$.

Proof. This is essentially an application of Lemma 7. See the Appendix. 
Comonad equations We prove that $\mathrm{d}_{!_{\mathbb{M}} X} \cdot \mathrm{p}_{X}=\mathrm{Id}_{!_{\mathbb{M}} X}$. Let $\left(\mu, \mu^{\prime}\right) \in !_{\mathbb{M}} X \times$ $!_{\mathbb{M}} X$. Assume first that $\left(\mu, \mu^{\prime}\right) \in \mathrm{d}_{!_{\mathbb{M}} X} \cdot \mathrm{p}_{X}$. Then we can find $M \in !_{\mathbb{M}} !_{\mathbb{M}} X$ such that $(\mu, M) \in \mathrm{p}_{X}$ and $\left(M, \mu^{\prime}\right) \in \mathrm{d}_{!_{\mathbb{M} X} X}$. This means that $M=\left[\mu^{\prime}\right]$ and hence $\Sigma(M)=\mu^{\prime}$, hence $\mu=\mu^{\prime}$. Conversely, for $\mu \in !_{\mathbb{M} X} X$ we have $(\mu,[\mu]) \in \mathrm{p}_{X}$, therefore $(\mu, \mu) \in \mathrm{d}_{!_{\mathbb{M}} X} \cdot \mathrm{p}_{X}$.

Next we prove that $!_{\mathbb{M}} d_{X} \cdot p_{X}=I_{!_{\mathbb{M}} X}$. Let $\left(\mu, \mu^{\prime}\right) \in !_{\mathbb{M}} d_{X} \cdot p_{X}$. Let $M \in$ $!_{\mathbb{M} !} !_{\mathbb{M}} X$ be such that $(\mu, M) \in \mathrm{p}_{X}$, that is $\Sigma(M)=\mu$, and $\left(M, \mu^{\prime}\right) \in !_{\mathbb{M}} \mathrm{d}_{X}$. Let $\sigma \in \mathbb{M}\left\langle!_{\mathbb{M}} X \times X\right\rangle$ be a witness of $\left(M, \mu^{\prime}\right)$ for $\mathrm{d}_{X}$. This means that $\mu^{\prime}(a)=$ $\sum_{\nu \in !_{\mathbb{M} X} X} \sigma(\nu, a)=\sigma([a], a)$ since $\operatorname{supp}(\sigma) \subseteq \mathrm{d}_{X}$, and that $M(\nu)=\sigma([a], a)$ if $\nu=[a]$, and $M(\nu)=0$ if $\# \nu \neq 1$. It follows that $\Sigma(M)=\sum_{\nu \in !_{\mathbb{M} X}} M(\nu) \nu=$ $\sum_{a \in X} \sigma([a], a)[a]=\mu^{\prime}$ and hence $\mu=\mu^{\prime}$. Conversely, one has $(\mu, \mu) \in !_{\mathbb{M}} \mathbf{d}_{X} \cdot \mathbf{p}_{X}$, because $M \in !_{\mathbb{M} !} !_{\mathbb{M}} X$ defined by $M(\nu)=\mu(a)$ if $\nu=[a]$ and $M(\nu)=0$ if $\# \nu \neq 0$ satisfies $(\mu, M) \in \mathrm{p}_{X}$ and $(M, \mu) \in !_{\mathbb{M}} \mathrm{d}_{X}$.

Lemma 9. Let $\mathcal{M} \in !_{\mathbb{M} !} !_{\mathbb{M} !} !_{\mathbb{M}} X$. Then $\Sigma(\Sigma(\mathcal{M}))=\sum_{N \in !_{\mathbb{M} !} X} \mathcal{M}(N) \Sigma(N)$.

Proof. We have

$$
\begin{aligned}
\Sigma(\Sigma(\mathcal{M})) & =\sum_{\nu \in !_{\mathbb{M}} X} \Sigma(\mathcal{M})(\nu) \nu \\
& =\sum_{\nu \in !_{\mathbb{M}} X}\left(\sum_{N \in !_{\mathbb{M} !} X} \mathcal{M}(N) N(\nu)\right) \nu \\
& =\sum_{N \in !_{\mathbb{M}} !_{\mathbb{M}} X} \mathcal{M}(N)\left(\sum_{\nu \in !_{\mathbb{M}} X} N(\nu) \nu\right)
\end{aligned}
$$

and we are done.

Lemma 10. The digging is natural, that is $\mathrm{p}_{!_{\mathbb{M}} X} \cdot \mathrm{p}_{X}=!_{\mathbb{M}} \mathrm{p}_{X} \cdot \mathrm{p}_{X}$, so let $(\mu, \mathcal{M}) \in$ $!_{\mathbb{M}} X \times !_{\mathbb{M} !} !_{\mathbb{M}} !_{\mathbb{M}} X$.

Proof. One applies Lemma 9, see the Appendix.

Fundamental isomorphism. One of the most important property of the exponential is that it maps cartesian products to tensor products. Combined with the monoidal closure of Rel, this property leads to the cartesian closure of the Kleisli category Rel!

Proposition 5. Given two sets $X_{1}$ and $X_{2}$, there is an natural bijection $\mathrm{n}_{X_{1}, X_{2}}$ : $!_{\mathbb{M}} X_{1} \otimes !_{\mathbb{M}} X_{2} \rightarrow !_{\mathbb{M}}\left(X_{1} \& X_{2}\right)$ and a bijection $\mathrm{n}_{0}: 1 \rightarrow !_{\mathbb{M}} \top$.

Structural morphisms. They are used for interpreting the structural rules of linear logic, associated with the exponentials. The weakening morphism is weak $_{X}: !_{\mathbb{M}} X \rightarrow 1$ is weak $_{X}=\{([], *)\}$. The contraction morphism is contr ${ }_{X}$ : $!_{\mathbb{M}} X \rightarrow !_{\mathbb{M}} X \otimes !_{\mathbb{M}} X$ is obtained by applying the $!_{\mathbb{M}}$ functor to the diagonal map $X \rightarrow X \& X$, so that $\operatorname{contr}_{X}=\left\{(\lambda+\rho,(\lambda, \rho)) \mid \lambda, \rho \in !_{\mathbb{M}} X\right\}$.

There are other equations to check for proving that we have defined a model of Linear Logic (see [Bie95]), the corresponding verifications are straightforward. 


\subsection{The Kleisli cartesian closed category}

The objects of the Kleisli category Rel! of the comonad "! ! ${ }_{\mathbb{M}}$ " are the sets, and $\operatorname{Rel}_{!}(X, Y)=\operatorname{Rel}\left(!_{\mathbb{M}} X, Y\right)$. Identity in this category is dereliction $\mathrm{d}_{X} \in$ $\operatorname{Rel}_{!}(X, X)$ and composition is defined as follows: let $R \in \operatorname{Rel}_{!}(X, Y)$ and $S \in \operatorname{Rel}_{!}(Y, Z)$, then

$$
S \circ R=S \cdot !_{\mathbb{M}} R \cdot \mathrm{p}_{X} \cdot
$$

We give a direct characterization of this composition law.

Proposition 6. Let $(\mu, c) \in !_{\mathbb{M}} X \times Y$, we have $(\mu, c) \in S \circ R$ iff there exist $b_{1}, \ldots, b_{n} \in Y$ (not necessarily distinct), $p_{1}, \ldots, p_{n} \in \mathbb{M}$ and $\mu_{1}, \ldots, \mu_{n} \in !_{\mathbb{M}} X$ such that

$$
\forall i \in\{1, \ldots, n\}\left(\mu_{i}, b_{i}\right) \in R, \quad\left(\sum_{i=1}^{n} p_{i}\left[b_{i}\right], c\right) \in S \quad \text { and } \quad \mu=\sum_{i=1}^{n} p_{i} \mu_{i} .
$$

Proof. Assume first that $(\mu, c) \in S \circ R$. Let $M \in !_{\mathbb{M} !} !_{\mathbb{M}} X$ such that $(\mu, M) \in \mathrm{p}_{X}$ and let $\nu \in !_{\mathbb{M}} Y$ be such that $(\nu, c) \in S$ and $(M, \nu) \in !_{\mathbb{M}} R$. We have $\Sigma(M)=\mu$. Let $\sigma \in \mathbb{M}\left\lfloor!_{\mathbb{M}} X \times Y\right\rangle$ be a witness of $(M, \nu)$ for $R$, and let $\left(\mu_{1}, b_{1}\right), \ldots,\left(\mu_{n}, b_{n}\right)$ be a repetition-free enumeration of the set $\operatorname{supp}(\sigma) \subseteq R$. Taking $p_{i}=\sigma\left(\mu_{i}, b_{i}\right)$, we have $\sum_{i=1}^{n} p_{i}\left[b_{i}\right]=\nu$ and $\sum_{i=1}^{n} p_{i}\left[\mu_{i}\right]=M$, and therefore $\mu=\sum_{i=1}^{n} p_{i} \mu_{i}$.

Assume conversely that $(\mu, c)$ satisfies the conditions stated in the proposition. Then we take $\nu=\sum_{i=1}^{n} p_{i}\left[b_{i}\right]$ and $M=\sum_{i=1}^{n} p_{i}\left[\mu_{i}\right]$. We have $(\nu, c) \in S$ and $(\mu, M) \in \mathrm{p}_{X}$ and we have just to check that $(M, \nu) \in !_{\mathbb{M}} R$. We define $\sigma=\sum_{i=1}^{n} p_{i}\left[\left(\mu_{i}, b_{i}\right)\right]$; this is a witness of $(M, \nu)$ for $R$, as easily checked.

We recall that the cartesian product of $X$ and $Y$ in this category is $X \&$ $Y$, with projections obtained by composing $\pi_{1}$ and $\pi_{2}$ with $\mathrm{d}_{X \& Y}$ in Rel. The function space of $X$ and $Y$ is $!_{\mathbb{M}} X \multimap Y$. Evaluation Ev $\in \operatorname{Rel}_{1}(X \&$ $\left.\left(!_{\mathbb{M}} X \multimap Y\right), Y\right) \simeq \operatorname{Rel}\left(!_{\mathbb{M}} X \otimes !_{\mathbb{M}}\left(!_{\mathbb{M}} X \multimap Y\right), Y\right)$ is

$$
\mathrm{Ev}=\left\{((\mu,[(\mu, b)]), b) \mid \mu \in !_{\mathbb{M}} X \text { and } b \in Y\right\} .
$$

Curryfication is defined as follows: let $R \in \operatorname{Rel}_{!}(Z \& X, Y) \simeq \operatorname{Rel}\left(!_{\mathbb{M}} Z \otimes\right.$ $\left.!_{\mathbb{M}} X, Y\right)$, then $\operatorname{Cur}(R)=\{(\pi,(\mu, b)) \mid((\pi, \mu), b) \in R\} \in \operatorname{Re}_{!}\left(Z, !_{\mathbb{M}} X \multimap Y\right)$.

Differential structure and the Taylor expansion. We sketch very briefly the differential structure of this model, which can be used for interpreting the differential lambda-calculus introduced in [ER03,Vau05], or the various resource lambda-calculi based on this kind of differential structures [ER08,Tra08].

We introduce first the codereliction morphism $\partial_{X} \in \operatorname{Rel}\left(X, !_{\mathbb{M}} X\right)$ by $\partial_{X}=$ $\{(a,[a]) \mid a \in X\}$. Naturality is proved exactly as the naturality of dereliction in 2.3. Coweakening $\operatorname{coweak}_{X} \in \operatorname{Rel}\left(1, !_{\mathbb{M}} X\right)$ and cocontraction cocontr ${ }_{X} \in$ $\operatorname{Rel}\left(!_{\mathbb{M}} X \otimes !_{\mathbb{M}} X, !_{\mathbb{M}} X\right)$ are obtained by applying the $!_{\mathbb{M}}$ functor to the empty morphism $\emptyset \in \operatorname{Rel}(\top, X)$ and to the "codiagonal" morphism $\pi_{1} \cup \pi_{2} \in \operatorname{Rel}(X \&$ $X, X)$, and by using the fundamental isomorphism. The equations involving dereliction and coweakening and cocontraction (see [ER06b,BCS06,Fio07]) are 
satisfied by naturality of dereliction. Similarly for the equations involving codereliction, weakening and contraction. One should check that the chain rule holds: this is a bit long to express, but the proof is a simple verification.

Using codereliction and cocontraction, one defines easily a morphism $\delta_{X} \in$ $\boldsymbol{\operatorname { R e l }}\left(!_{\mathbb{M}} X \otimes X, !_{\mathbb{M}} X\right)$, with $\delta_{X}=\{(\mu, a, \mu+[a])\}$. Given $R \in \operatorname{Rel}(X, Y)=$ $\operatorname{Rel}\left(!_{\mathbb{M}} X, Y\right)$, one can define $R^{\prime} \in \operatorname{Rel}_{!}(X, X \multimap Y$ ) (by linear curryfication of $\left.R \cdot \delta_{X}\right)$ which can be considered as the first derivative of $R$ : this operation satisfies all the usual rules of differential calculus (linearity, Leibniz rule, chain rule etc). Iterating this operation and using also dereliction and contraction, one can define the Taylor expansion of $R$ as the infinite union $\bigcup_{n \in \mathbb{N}}^{\infty} R_{n}$ where, for each $n \in \mathbb{N} \subseteq \mathbb{M}$ the morphism $R_{n} \in \operatorname{Rel}_{!}(X, Y)$ is simply $\{(\mu, a) \in R \mid \# \mu=n\}$. So if $\mathbb{M}$ has infinite elements, it is not true in general that $R$ coincides with its Taylor expansion. As an example, let $\omega$ be an infinite element of $\mathbb{M}$ and take $R=\{(\omega[*], *)\} \in \mathbf{R e l}_{!}(1,1)$. Then $R_{n}=\emptyset$ for all $n \in \mathbb{N}$.

So we have defined a model of differential linear logic which does not satisfy the Taylor formula.

\section{Graph models in Rel}

Graph models [Bar84] have been isolated by Scott and Engeler in the continuous semantics. We develop here a similar construction, in the relational semantics. Let $A$ be a non-empty set whose elements will be called atoms, and are not pairs. Let $\iota: A \rightarrow\left(!_{\mathbb{M}} A \multimap A\right)$ be a partial injective map.

We define a sequence $\left(D_{n}^{\iota}\right)_{n \in \mathbb{N}}$ of sets as follows: $D_{0}^{\iota}=A$ and $D_{n+1}^{\iota}=D_{n}^{\iota} \cup$ $\left(\left(!_{\mathbb{M}} D_{n}^{\iota} \multimap D_{n}^{\iota}\right) \backslash \iota(A)\right)$. This sequence is monotone, and we set $D^{\iota}=\bigcup_{n \in \mathbb{N}} D_{n}^{\iota}$. We have $!_{\mathbb{M}} D^{\iota} \multimap D^{\iota}=\bigcup_{n \in \mathbb{N}}\left(!_{\mathbb{M}} D_{n}^{\iota} \multimap D_{n}^{\iota}\right)$.

We define a function $\varphi: D^{\iota} \rightarrow\left(!_{\mathbb{M}} D^{\iota} \multimap D^{\iota}\right)$ by

$$
\varphi(\alpha)= \begin{cases}\iota(a) & \text { if } \alpha=a \in A \\ \alpha & \text { if } \alpha \notin A\end{cases}
$$

and a function $\psi:\left(!_{\mathbb{M}} D^{\iota} \multimap D^{\iota}\right) \rightarrow D^{\iota}$ by

$$
\psi(\mu, \alpha)= \begin{cases}a & \text { if }(\mu, \alpha)=\iota(a) \text { where } a \in A \\ (\mu, \alpha) & \text { if }(\mu, \alpha) \notin \iota(A) .\end{cases}
$$

This definition makes sense because $\iota$ is injective, and because, if $(\mu, \alpha) \in$ $\left(!_{\mathbb{M}} D_{n}^{\iota} \multimap D_{n}^{\iota}\right) \backslash \iota(A)$, then $(\mu, \alpha) \in D_{n+1}^{\iota} \subseteq D^{\iota}$. Let $(\mu, \alpha) \in !_{\mathbb{M}} D^{\iota} \multimap D^{\iota}$. If $(\mu, \alpha) \in \iota(A)$, let $a$ be the unique element of $A$ such that $\iota(a)=(\mu, \alpha)$. We have $\varphi(\psi(\mu, \alpha))=\varphi(a)=\iota(a)=(\mu, \alpha)$. If $(\mu, \alpha) \notin \iota(A)$, we have $\varphi(\psi(\mu, \alpha))=$ $\varphi(\mu, \alpha)=(\mu, \alpha)$ because $(\mu, \alpha) \notin A$, since no element of $A$ is a pair.

It is clear that $\varphi \circ \psi=\mathrm{Id}$. We define two morphisms App $=\{([\alpha], \varphi(\alpha)) \mid \alpha \in$ $\left.D^{\iota}\right\} \in \operatorname{Rel}_{!}\left(D^{\iota}, !_{\mathbb{M}} D^{\iota} \multimap D^{\iota}\right)$ and Lam $=\left\{([(\mu, \alpha)], \psi(\mu, \alpha)) \mid(\mu, \alpha) \in !_{\mathbb{M}} D^{\iota} \multimap\right.$ $\left.D^{\iota}\right\} \in \operatorname{Rel}_{!}\left(!_{\mathbb{M}} D^{\iota} \multimap D^{\iota}, D^{\iota}\right)$. Then we have App $\circ$ Lam $=\operatorname{Id}_{!_{\mathbb{M}} D^{\iota} \multimap D^{\iota}}$, so that $D^{\iota}$ is a reflexive object in $\mathbf{R e l}_{\text {! }}$, whatever be the choice of the multiplicity semi-ring $\mathbb{M}$. 


\subsection{Interpreting terms}

Given a lambda-term $M$ and a repetition-free list of variables $\boldsymbol{x}=\left(x_{1}, \ldots, x_{n}\right)$ which contains all free variables of $M$, the interpretation $[M]_{\boldsymbol{x}} \in \mathbf{R e l}_{!}\left(D^{\iota n}, D^{\iota}\right)$ (where $D^{\iota n}$ is the cartesian product of $D^{\iota}$ with itself, $n$ times) is defined by induction on $M$ as follows

- $\left[x_{i}\right]_{\boldsymbol{x}}=\pi_{i}$ (the $i$ th projection from $D^{\iota n}$ to $D$ )

- $[\lambda x N]_{\boldsymbol{x}}=\operatorname{Lam} \circ \operatorname{Cur}\left([M]_{\boldsymbol{x}, x}\right)$

- $[(N) P]_{\boldsymbol{x}}=\mathrm{Ev} \circ\left\langle\operatorname{App} \circ[N]_{\boldsymbol{x}},[P]_{\boldsymbol{x}}\right\rangle$

Using the cartesian closeness of $\mathbf{R e l}_{!}$and the fact that App $\circ$ Lam $=\operatorname{Id}_{!_{\mathbb{M}} D^{\iota} \multimap D^{\iota}}$, one proves that if $M$ and $M^{\prime}$ are beta-equivalent, and $\boldsymbol{x}$ is a repetition-free list of variables which contain all the free variables of $M$ and $M^{\prime}$, one has $[M]_{\boldsymbol{x}}=\left[M^{\prime}\right]_{\boldsymbol{x}}$. This requires to prove first a substitution lemma, see [AC98].

We present now this interpretation as a typing system (a variation of de Carvalhos's system R [DC08]). A type is an element of $D^{\iota}$. Given $\mu \in !_{\mathbb{M}} D^{\iota}$ and $\alpha \in D^{\iota}$, we set $\mu \rightarrow \alpha=\psi(\mu, \alpha)$. A typing context is a finite partial function from variables to $!_{\mathbb{M}} D^{\iota}$. If $\Gamma_{1}, \ldots, \Gamma_{k}$ are contexts with the same domain and $p_{1}, \ldots, p_{k} \in \mathbb{M}$, the sum $\sum_{i=1}^{k} p_{i} \Gamma_{i}$ is defined pointwise (using the addition of $\left.!_{\mathbb{M}} D^{\iota}\right)$. The typing rules are

$$
\begin{array}{cc}
\hline x_{1}:[], \ldots, x_{n}:[], x:[\alpha] \vdash x: \alpha & \frac{\Gamma, x: \mu \vdash M: \alpha}{\Gamma \vdash \lambda x M: \mu \rightarrow \alpha} \\
\frac{\Gamma \vdash M:\left(\sum_{i=1}^{n} p_{i}\left[\beta_{i}\right]\right) \rightarrow \alpha}{\Gamma+\sum_{i=1}^{n} p_{i} \Gamma_{i} \vdash(M) N: \alpha} & \forall i \in \bar{n} \quad \Gamma_{i} \vdash N: \beta_{i} \\
\hline
\end{array}
$$

In the last rule, all contexts involved must have same domain.

Proposition 7. The judgment $\Gamma \vdash M: \alpha$ is derivable iff $\left(\Gamma\left(x_{1}\right), \ldots, \Gamma\left(x_{n}\right), \alpha\right) \in$ $[M]_{\boldsymbol{x}}$ where $\boldsymbol{x}=\left(x_{1}, \ldots, x_{n}\right)$ is a repetition-free enumeration of the domain of $\Gamma$, which is assumed to contain all the free variables of $M$.

Proof. Straightforward induction on the judgment.

We take for $\mathbb{M}$ a multiplicity semi-ring which contains an element $\omega$ such that $\omega+1=\omega$. Let $A=\{a\}, \iota: A \rightarrow\left(!_{\mathbb{M}} A \multimap A\right)$ be defined by $\iota(a)=(\omega[a], a)$, so that $(\omega[a] \rightarrow a)=a$. Let $\Omega=(\delta) \delta$ where $\delta=\lambda x(x) x$.

Proposition 8. In the model $D^{\iota}$, we have $[\Omega]=\{a\}$.

Proof. We have the following deduction tree (we have inserted in this tree the equations between types or $\mathbb{M}$-multisets of types that we use)

$$
\frac{x:[a] \vdash x: a=\omega[a] \rightarrow a \quad x:[a] \vdash x: a}{\frac{x:[a]+\omega[a]=\omega[a] \vdash(x) x: a}{\vdash \lambda x(x) x: \omega[a] \rightarrow a}} \quad \frac{\text { (same derivation) }}{\vdash(\lambda x(x) x) \lambda x(x) x: a}
$$


Therefore $a \in[\Omega]$.

Conversely, let $\alpha \in D^{\iota}$ and assume that $\vdash \Omega: \alpha$. There must exist $\mu \in !_{\mathbb{M}} D^{\iota}$ such that $\vdash \delta: \mu \rightarrow \alpha$ and $\forall \beta \in \operatorname{supp}(\mu) \vdash \delta: \beta$. Form the first of these two judgments we get $x: \mu \vdash(x) x: \alpha$ and hence there must exist $\nu \in !_{\mathbb{M}} D^{\iota}$ such that $\mu=\nu+[\nu \rightarrow \alpha]$. From the second judgment we get $\vdash \delta: \nu \rightarrow \alpha$ and $\forall \beta \in \operatorname{supp}(\nu) \vdash \delta: \beta$. Iterating this process, we build a sequence $\left(\mu_{i}\right)_{i=1}^{\infty}$ of elements of $!_{\mathbb{M}} D^{\iota}$ such that $\vdash \delta: \mu_{i} \rightarrow \alpha, \forall \beta \in \operatorname{supp}\left(\mu_{i}\right) \vdash \delta: \beta$ and $\mu_{i}=$ $\mu_{i+1}+\left[\mu_{i+1} \rightarrow \alpha\right]$ for all $i$. Let $\beta_{i}=\mu_{i} \rightarrow \alpha$, it follows that $\forall i \beta_{i} \in \operatorname{supp}\left(\mu_{1}\right)$ and since $\operatorname{supp}\left(\mu_{1}\right)$ is finite, we can find $i$ and $n>0$ such that $\beta_{i+n}=\beta_{i}$. We have $\beta_{i}=\left(\mu_{i} \rightarrow \alpha\right)=\left(\left(\mu_{i+1}+\left[\beta_{i+1}\right]\right) \rightarrow \alpha\right)=\cdots=\left(\left(\mu_{i+n}+\left[\beta_{i+1}\right]+\cdots+\left[\beta_{i+n}\right]\right) \rightarrow \alpha\right)$ and since $\beta_{i+n}=\left(\mu_{i+n} \rightarrow \alpha\right)$, we get $\mu_{i+n}=\mu_{i+n}+\left[\beta_{i+1}\right]+\cdots+\left[\beta_{i+n}\right]$ (because $\psi$ is injective) and hence $\beta_{i+n} \in \operatorname{supp}\left(\mu_{i+n}\right)$. But $\beta_{i+n}=\left(\mu_{i+n} \rightarrow \alpha\right)$ and hence we must have $\beta_{i+n}=a$. Indeed, if $\beta_{i+n} \notin A$ then we have $\beta_{i+n}=\left(\mu_{i+n}, \alpha\right)$ and, if $k$ is the least integer such that $\beta_{i+n} \in D_{k}^{\iota}$, we have $k>0$ and $\beta \in D_{k-1}^{\iota}$ for all $\beta \in \operatorname{supp}\left(\mu_{i+n}\right)$. This is impossible since $\beta_{i+n} \in \operatorname{supp}\left(\mu_{i+n}\right)$. Since $\left(\mu_{i+n} \rightarrow\right.$ $\alpha)=a$, we have $\alpha=a$ and we are done.

Since $([] \rightarrow a) \in[\lambda y \Omega]$ and $a \neq([] \rightarrow a)$, we have found two unsolvable terms (namely $\Omega$ and $\lambda y \Omega$ ) with distinct interpretations in $D^{\iota}$ and hence this model is not sensible.

\section{Conclusion}

We have introduced the concept of multiplicity semi-ring, which can be used for generalizing the standard exponential construction of the relational model of Linear Logic. Such a semi-ring must contain $\mathbb{N}$ as a sub-semi-ring but can also have infinite elements $\omega$ such that $\omega+1=\omega$. In that case, the corresponding model of Linear Logic is a model of the differential lambda-calculus which does not satisfy the Taylor formula, and it is possible to build non sensible models of the lambda-calculus in the corresponding Kleisli cartesian closed category. This shows that models of the pure differential lambda-calculus can have non sensible theories and provides a new way of building models of the pure lambda-calculus where non termination is taking into account in a quantitative way by means of these infinite multiplicities.

\section{References}

[AC98] Roberto Amadio and Pierre-Louis Curien. Domains and lambda-calculi, volume 46 of Cambridge Tracts in Theoretical Computer Science. Cambridge University Press, 1998.

[Bar84] Henk Barendregt. The Lambda Calculus, volume 103 of Studies in Logic and the Foundations of Mathematics. North Holland, 1984.

[BCS06] Richard Blute, Robin Cockett, and Robert Seely. Differential categories. Mathematical Structures in Computer Science, 16(6):1049-1083, 2006. 
[BEM07] Antonio Bucciarelli, Thomas Ehrhard, and Giulio Manzonetto. Not enough points is enough. In Proceedings of the 21st Annual Conference of the European Association for Computer Science Logic (CSL'O7), Lecture Notes in Computer Science. Springer-Verlag, September 2007.

[BEM09] Antonio Bucciarelli, Thomas Ehrhard, and Giulio Manzonetto. A relational model of a parallel and non-deterministic lambda-calculus. In Sergei N. Artëmov and Anil Nerode, editors, LFCS, volume 5407 of Lecture Notes in Computer Science, pages 107-121. Springer, 2009.

[Bie95] Gavin Bierman. What is a categorical model of intuitionistic linear logic? In Mariangiola Dezani-Ciancaglini and Gordon D. Plotkin, editors, Proceedings of the second Typed Lambda-Calculi and Applications conference, volume 902 of Lecture Notes in Computer Science, pages 73-93. Springer-Verlag, 1995.

[DC08] Daniel De Carvalho. Execution Time of $\lambda$-Terms via Denotational Semantics and Intersection Types. Research Report RR-6638, INRIA, 2008.

[EL09] Thomas Ehrhard and Olivier Laurent. Interpreting a finitary pi-calculus in differential interaction nets. Information and Computation, 2009. To appear.

[ER03] Thomas Ehrhard and Laurent Regnier. The differential lambda-calculus. Theoretical Computer Science, 309(1-3):1-41, 2003.

[ER04] Thomas Ehrhard and Laurent Regnier. Differential interaction nets. In Proceedings of WoLLIC'04, volume 103 of Electronic Notes in Theoretical Computer Science, pages 35-74. Elsevier Science, 2004.

[ER06a] Thomas Ehrhard and Laurent Regnier. Böhm trees, Krivine machine and the Taylor expansion of ordinary lambda-terms. In Arnold Beckmann, Ulrich Berger, Benedikt Löwe, and John V. Tucker, editors, Logical Approaches to Computational Barriers, volume 3988 of Lecture Notes in Computer Science, pages 186-197. Springer-Verlag, 2006.

[ER06b] Thomas Ehrhard and Laurent Regnier. Differential interaction nets. Theoretical Computer Science, 364(2):166-195, 2006.

[ER08] Thomas Ehrhard and Laurent Regnier. Uniformity and the Taylor expansion of ordinary lambda-terms. Theoretical Computer Science, 403(2-3):347-372, 2008.

[Fio07] Marcelo P. Fiore. Differential structure in models of multiplicative biadditive intuitionistic linear logic. In Simona Ronchi Della Rocca, editor, TLCA, volume 4583 of Lecture Notes in Computer Science, pages 163-177. Springer, 2007.

[MS09] Giulo Manzonetto and Antonino Salibra. Applying universal algebra to lambda calculus. Journal of Logic and Computation, 2009. To appear.

[PS98] Don Pigozzi and Antonino Salibra. Lambda Abstraction Algebras: Coordinatizing Models of Lambda Calculus. Fundamenta Informaticae, 33(2):149-200, 1998.

[Tra08] Paolo Tranquilli. Intuitionistic Differential Nets and Lambda-Calculus. Theoretical Computer Science, 2008. To appear.

[Vau05] Lionel Vaux. The differential lambda-mu calculus. Theoretical Computer Science, 379(1-2):166-209, 2005.

\section{Appendix: omitted proofs}

Proof of Lemma 1. Assume first that $l=2$ and let us prove the result by induction on $r$. For $r=1$, one takes $s_{11}=n_{1}$ and $s_{21}=n_{2}$. Assume that the 
property holds for $r$ and let us prove it for $r+1$. Let $q_{1}=\sum_{j=1}^{r} p_{j}$ and $q_{2}=p_{r+1}$. Then, applying Condition (MS3), we can find $t_{i k} \in M$ for $i=1,2$ and $k=1,2$ such that $n_{1}=t_{11}+t_{12}, n_{2}=t_{21}+t_{22}, q_{1}=t_{11}+t_{21}$ and $q_{2}=t_{12}+t_{22}$. By inductive hypothesis we can find a family $\left(u_{i j}\right)_{i=1, j=1}^{2, r}$ such that $t_{11}=\sum_{j=1}^{r} u_{1 j}$, $t_{21}=\sum_{j=1}^{r} u_{2 j}$ and $p_{j}=u_{1 j}+u_{2 j}$ for $j=1, \ldots, r$. Then we define $\left(s_{i j}\right)_{i=1, j=1}^{2, r+1}$ by setting $s_{i j}=u_{i j}$ for $i=1,2$ and $j=1, \ldots, r$, and $s_{i, r+1}=t_{i 2}$ for $i=1,2$.

Now we prove the result for an arbitrary value of $l$, by induction on this parameter. For $l=1$, we set $s_{1 j}=p_{j}$ for $j=1, \ldots, r$. Assume that the result holds for $l$ and let us prove it for $l+1$. Let $m_{1}=\sum_{i=1}^{l} n_{i}$ and $m_{2}=n_{l+1}$. We have $m_{1}+m_{2}=\sum_{j=1}^{r} p_{j}$ so we can apply the property that we have just proved. Let $\left(t_{k j}\right)_{k=1, j=1}^{2, r}$ be a family of elements of $M$ such that $m_{k}=\sum_{j=1}^{r} t_{k j}$ for $k=1,2$ and $p_{j}=t_{1 j}+t_{2 j}$ for $j=1, \ldots, r$. By inductive hypothesis, we can find a family $\left(u_{i j}\right)_{i=1, j=1}^{l, r}$ such that $n_{i}=\sum_{j=1}^{r} u_{i j}$ for $i=1, \ldots, l$ and $p_{j}=\sum_{i=1}^{l} u_{i j}$. Then we define a family $\left(s_{i j}\right)_{i=1, j=1}^{l+1, r}$ by setting $s_{i j}=u_{i j}$ for $i=1, \ldots, l$ and $j=1, \ldots, r$, and $s_{l+1, j}=t_{2 j}$ for $j=1, \ldots, r$, and we are done.

Proof of Lemma 2. By induction on $k$. For $k=1$, one has $l=1$, and one takes $p_{1}=p$ and $m_{11}=m$.

Assume that the result holds for $k$ (and let $l=2^{k-1}$ ) and let us prove it for $k+$ 1. Let $n_{1}, \ldots, n_{k+1}, m, p \in M$ with $n_{1}+\cdots+n_{k+1}=m p$. By inductive hypothesis, we can find $\left(p_{j}\right)_{j=1}^{l} \in M$ and $\left(m_{i j}\right)_{i=1, j=1}^{k, l}$ with the following properties

- $p_{1}+\cdots+p_{l}=p$

- $m_{1 j}+\cdots+m_{k j}=m$ for $j=1, \ldots, l$

- $m_{i 1} p_{1}+\cdots+m_{i l} p_{l}=n_{i}$ for $i=1, \ldots, k-1$

- and $m_{k 1} p_{1}+\cdots+m_{k l} p_{l}=n_{k}+n_{k+1}$.

By Lemma 1, we can find a family $\left(r_{j h}\right)_{j=1, h=0}^{l, 1}$ of elements of $M$ such that $n_{k+h}=r_{1 h}+\cdots+r_{l h}$ for $h=0,1$ and $\forall j \in \bar{l} m_{k j} p_{j}=r_{j 0}+r_{j 1}$. By Condition (MS4), for each $j \in \bar{l}$, we can find $p_{j 1}, p_{j 2} \in M$ with $p_{j 1}+p_{j 2}=p_{j}$ and a family $\left(s_{j u h}\right)_{u=1, h=0}^{2,1}$ of elements of $M$ such that $m_{k j}=s_{j 10}+s_{j 11}=s_{j 20}+s_{j 21}$ and $r_{j h}=s_{j 1 h} p_{j 1}+s_{j 2 h} p_{j 2}$ for each $j \in \bar{l}$ and $h=0,1$. For $i \in \overline{k-1}$ we have

$$
n_{i}=m_{i 1} p_{11}+m_{i 1} p_{12}+\cdots+m_{i l} p_{l 1}+m_{i l} p_{l 2}
$$

and for $h=0,1$, we have

$$
\begin{aligned}
n_{k+h} & =r_{1 h}+\cdots+r_{l h} \\
& =s_{11 h} p_{11}+s_{12 h} p_{12}+\cdots+s_{l 1 h} p_{l 1}+s_{l 2 h} p_{l 2} .
\end{aligned}
$$

Let us define a family $\left(p_{j^{\prime}}^{\prime}\right)_{j^{\prime}=1}^{2 l}$ of elements of $M$ by setting $p_{1}^{\prime}=p_{11}, p_{2}^{\prime}=$ $p_{12}, \ldots, p_{2 l-1}^{\prime}=p_{l 1}, p_{2 l}^{\prime}=p_{l 2}$ and let use define a family $\left(m_{i j^{\prime}}^{\prime}\right)_{i=1, j^{\prime}=1}^{k+1,2 l}$ by setting $m_{i, 2 j-1}^{\prime}=m_{i, 2 j}^{\prime}=m_{i j}$ for $i \in \overline{k-1}$ and $j \in \bar{l}$, and by $m_{k+h, 2 j-1}^{\prime}=s_{j 1 h}$ and $m_{k+h, 2 j}^{\prime}=s_{j 2 h}$ for $j \in \bar{l}$ and $h=0,1$. With these definitions, we have 
- $p_{1}^{\prime}+\cdots+p_{2 l}^{\prime}=p_{1}+\cdots+p_{l}=p$

- $m_{1,2 j-1}^{\prime}+\cdots+m_{k+1,2 j-1}^{\prime}=m_{1, j}+\cdots+m_{k-1, j}+s_{j 10}+s_{j 11}=m_{1 j}+\cdots+m_{k j}=$ $m$ for $j=1, \ldots, l$

- $m_{1,2 j}^{\prime}+\cdots+m_{k+1,2 j}^{\prime}=m_{1, j}+\cdots+m_{k-1, j}+s_{j 20}+s_{j 21}=m_{1 j}+\cdots+m_{k j}=m$ for $j=1, \ldots, l$

- $m_{i, 1}^{\prime} p_{1}^{\prime}+\cdots+m_{i, 2 l}^{\prime} p_{2 l}^{\prime}=n_{i}$ for $i=1, \ldots, k+1$

and the lemma is proved.

Proof of Proposition 1. One defines a map $f: \mathbb{N} \rightarrow M$ by induction on natural numbers by $f(0)=0$ and $f(n+1)=f(n)+1$, that is $f(n)=\sum_{i=1}^{n} 1$; we denote this sum as $n \cdot 1$. This map is a semi-ring morphism as easily checked, by induction on natural numbers again. We prove that $f$ is injective, so let $p \in \mathbb{N}$ and let us prove that $f(n)=f(n+p) \Rightarrow p=0$ by induction on $n$. For $n=0$, assume that $p \cdot 1=0$. Applying Condition (MS1) we get easily $p=0$ (by induction on $p$ actually). Assume now that $(n+1+p) \cdot 1=(n+1) \cdot 1$, that is $(n+p) \cdot 1+1=n \cdot 1+1$. By Condition (MS3), one can find $r_{11}, r_{12}, r_{21}, r_{22} \in \mathbb{M}$ such that $n+p=r_{11}+r_{12}, 1=r_{21}+r_{22}, n=r_{11}+r_{21}$ and $1=r_{12}+r_{22}$. By Condition (MS2), there are two cases to consider: either $r_{22}=1$, and in that case $r_{21}=r_{12}=0$, or $r_{22}=0$, and in that case $r_{21}=r_{12}=1$. In both cases we have $n+p=n$ and hence $p=0$ by inductive hypothesis.

Proof of Proposition 2. Let us check Condition (MS3), so let $n_{1}, n_{2}, p_{1}, p_{2} \in \mathbb{N}$ be such that $n_{1}+n_{2}=p_{1}+p_{2}$ and let $q$ be this common value. Pick arbitrarily sets $I_{1}, I_{2}, J_{1}, J_{2} \subseteq \bar{q}$ of respective cardinality $n_{1}, n_{2}, p_{1}$ and $p_{2}$. It suffices to take $r_{i j}=\#\left(I_{i} \cap J_{j}\right)$.

We prove now Condition (MS4). We apply Euclidian division by $p$ and we get $n_{1}=q_{1} p+r_{1}$ and $n_{2}=q_{2} p+r_{2}$ where $r_{1}, r_{2}<p$. We have $r_{1}+r_{2}=$ $p\left(m-q_{1}-q_{2}\right)$, and since $r_{1}, r_{2}<p$, we must have $m-q_{1}-q_{2}=0$ or $m-$ $q_{1}-q_{2}=1$. In the first case we have $r_{1}=r_{2}=0$. Pick $p_{1}, p_{2} \in \mathbb{N}$ such that $p_{1}+p_{2}=p$. Set $m_{11}=m_{12}=q_{1}$ and $m_{21}=m_{22}=q_{2}$. Then we have $m_{11}+m_{21}=m_{12}+m_{22}=m, p_{1} m_{11}+p_{2} m_{12}=p_{1} q_{1}+p_{2} q_{1}=p q_{1}=n_{1}$ and $p_{1} m_{21}+p_{2} m_{22}=p_{1} q_{2}+p_{2} q_{2}=p q_{2}=n_{2}$ as required. Assume now that $m-q_{1}-q_{2}=1$. We set $p_{1}=r_{1}, p_{2}=r_{2}, m_{11}=q_{1}+1, m_{12}=q_{1}, m_{21}=q_{2}$ and $m_{22}=q_{2}+1$. We have $m_{11}+m_{21}=m_{12}+m_{22}=q_{1}+q_{2}+1=m$. Next we have $p_{1} m_{11}+p_{2} m_{12}=r_{1}\left(q_{1}+1\right)+r_{2} q_{1}=\left(r_{1}+r_{2}\right) q_{1}+r_{1}=p q_{1}+r_{1}=n_{1}$. Similarly we have $p_{1} m_{21}+p_{2} m_{22}=n_{2}$, as required.

Proof of Proposition 3. We check Condition (MS3), so assume that $n_{1}+n_{2}=p_{1}+$ $p_{2}=q$. If $q \neq \omega$, then we have $n_{i}, p_{j} \in \mathbb{N}$ for each $i, j$ and we use Condition (MS3) for $\mathbb{N}$. Assume that $q=\omega$. Without loss of generality we can assume that $n_{1}=$ $p_{1}=\omega$. We can take $r_{11}=\omega, r_{22}=0, r_{12}=p_{2}$ and $r_{21}=n_{2}$. Last we check Condition (MS3), so assume that $p m=n_{1}+n_{2}=q$. Assume first that $q \in \mathbb{N}$. If $q \neq 0$, we know that $p, m, n_{1}, n_{2} \in \mathbb{N}$ and we can use Condition (MS4) in $\mathbb{N}$. If $q=0$, then $n_{1}=n_{2}=0$ and we must have $m=0$ or $p=0$. If $p=\omega$ and 
$m=0$ then we can take $p_{1}=\omega, p_{2}=0, m_{11}=m_{12}=m_{21}=m_{22}=0$. If $p=0$ and $m=\omega$, we take $p_{1}=p_{2}=0, m_{11}=m_{21}=\omega$ and $m_{12}=m_{22}=0$. We are left with the case were $q=\omega$. Without loss of generality we can assume that $n_{1}=\omega$, and of course we must have $m \neq 0$ and $p \neq 0$. Assume first that $p=\omega$. Then we can take $p_{1}=\omega, p_{2}=n_{2}, m_{11}=m, m_{21}=0, m_{12}=m^{\prime}$ such that $m^{\prime}+1=m$ and $m_{22}=1$. Assume last that $m=\omega$. Then we can take $p_{1}=p^{\prime}$ with $p^{\prime}+1=p, p_{2}=1, m_{11}=\omega, m_{21}=\omega, m_{12}=\omega$ and $m_{22}=n_{2}$.

Proof of Proposition 4. A simple case analysis shows that this addition is associative, and it is obvious that it is commutative. Distributivity is easily checked as well, so that we have defined a semi-ring. Observe that $\omega+1=\omega$, but that $\omega+\omega=2 \omega \neq \omega$ and actually, unlike $\overline{\mathbb{N}}$, this semi-ring has no element $n$ such that $n+n=n$ (apart from 0 of course).

Let us check the splitting property, so assume that $n_{1} \omega^{d_{1}}+n_{2} \omega^{d_{2}}=p_{1} \omega^{e_{1}}+$ $p_{2} \omega^{e_{2}}$ and let us build a family $\left(q_{i j} \omega^{f_{i j}}\right)_{i=1, j=1}^{2,2}$. If $d_{1}=d_{2}$ and $e_{1}=e_{2}$, we are reduced to the splitting property of $\mathbb{N}$. If $d_{1}=d_{2}$ and $e_{1}>e_{2}$, then we have $\left(n_{1}+n_{2}\right) \omega^{d_{1}}=p_{1} \omega^{e_{1}}$. We can set for instance $q_{11} \omega^{f_{11}}=n_{1} \omega^{d_{1}}, q_{12} \omega^{f_{12}}=$ $p_{2} \omega^{e_{2}}, q_{21} \omega^{f_{21}}=n_{2} \omega^{d_{1}}$ and $q_{22} \omega^{f_{22}}=0$. Then we have $q_{11} \omega^{f_{11}}+q_{12} \omega^{f_{12}}=$ $n_{1} \omega^{d_{1}}+p_{2} \omega^{e_{2}}=n_{1} \omega^{d_{1}}, q_{21} \omega^{f_{21}}+q_{22} \omega^{f_{22}}=n_{2} \omega^{d_{1}}, q_{11} \omega^{f_{11}}+q_{21} \omega^{f_{21}}=n_{1} \omega^{d_{1}}+$ $n_{2} \omega^{d_{1}}=p_{1} \omega^{e_{1}}$ and $q_{12} \omega^{f_{12}}+q_{22} \omega^{f_{22}}=p_{2} \omega^{e_{2}}$. The last case to consider (up to commutativity of addition) is $d_{1}>d_{2}$ and $e_{1}>e_{2}$. Then we know that $n_{1} \omega^{d_{1}}=p_{1} \omega^{e_{1}}$. We can set $q_{11} \omega^{f_{11}}=n_{1} \omega^{d_{1}}, q_{12} \omega^{f_{12}}=p_{2} \omega^{e_{2}}, q_{21} \omega^{f_{21}}=n_{2} \omega^{d_{2}}$ and $q_{22} \omega^{f_{22}}=0$.

Let us check Condition (MS4), so assume that $m \omega^{c} p \omega^{e}=n_{1} \omega^{d_{1}}+n_{2} \omega^{d_{2}}$. If $d_{1}=d_{2}$ we are reduced to Condition (MS4) in $\mathbb{N}$, so assume that $d_{2}<d_{1}$ (and of course $n_{1} \neq 0$ and $n_{2} \neq 0$ ). So we have $p m \omega^{e+c}=n_{1} \omega^{d_{1}}$. Our goal is to find $\left(p_{j} \omega^{e_{j}}\right)_{j=1}^{2}$ and $\left(m_{i j} \omega^{c_{i j}}\right)_{i=1, j=1}^{2,2}$ with $p_{1} \omega^{e_{1}}+p_{2} \omega^{e_{2}}=p \omega^{e}, p_{1} \omega^{e_{1}} m_{i 1} \omega^{c_{i 1}}+$ $p_{2} \omega^{e_{2}} m_{i 2} \omega^{c_{i 2}}=n_{i} \omega^{d_{i}}$ for $i=1,2$ and $m_{1 j} \omega^{c_{1 j}}+m_{2 j} \omega^{c_{2 j}}=m \omega^{c}$ for $j=1,2$. We consider several cases. For the two first cases, we set $p_{1} \omega^{e_{1}}=p \omega^{e}, m_{11} \omega^{c_{11}}=$ $m \omega^{c}$ and $m_{21} \omega^{c_{21}}=0$, so that $m_{11} \omega^{c_{11}}+m_{21} \omega^{c_{21}}=m \omega^{c}$ holds trivially.

- Assume first that $c \leq d_{2}$. We set $p_{2} \omega^{e_{2}}=n_{2} \omega^{d_{2}-c}, m_{22} \omega^{c_{22}}=\omega^{c}$ and $m_{12} \omega^{c_{12}}=(m-1) \omega^{c}$. We have $e+c=d_{1}>d_{2}$ and hence $e_{1}=e>$ $d_{2}-c=e_{2}$ so that $p_{1} \omega^{e_{1}}+p_{2} \omega^{e_{2}}=p \omega^{e}$. Next we have $m_{12} \omega^{c_{12}}+m_{22} \omega^{c_{22}}=$ $(m-1) \omega^{c}+\omega^{c}=m \omega^{c}$. And $p_{1} \omega^{e_{1}} m_{11} \omega^{c_{11}}+p_{2} \omega^{e_{2}} m_{12} \omega^{c_{12}}=p m \omega^{e+c}+$ $n_{2}(m-1) \omega^{d_{2}}=p m \omega^{d_{1}}=n_{1} \omega^{d_{1}}$ since $e+c=d_{1}>d_{2}$. Last we have $p_{1} \omega^{e_{1}} m_{21} \omega^{c_{21}}+p_{2} \omega^{e_{2}} m_{22} \omega^{c_{22}}=0+n_{2} \omega^{d_{2}-c} \omega^{c}=n_{2} \omega^{d_{2}}$.

- Assume now that $c>d_{2}$ and that $e>0$. We set $p_{2} \omega^{e_{2}}=n_{2}\left(\right.$ so $\left.e_{2}=0\right)$, $m_{22} \omega^{c_{22}}=\omega^{d_{2}}, m_{12} \omega^{c_{12}}=m \omega^{c}$. Then we have $p_{1} \omega^{e_{1}}+p_{2} \omega^{e_{2}}=p \omega^{e}+n_{2}=$ $p \omega^{e}$ since $e>0$. Also $m_{12} \omega^{c_{12}}+m_{22} \omega^{c_{22}}=m \omega^{c}+\omega^{d_{2}}=m \omega^{c}$ since we have assumed that $c>d_{2}$. Next we have $p_{1} \omega^{e_{1}} m_{11} \omega^{c_{11}}+p_{2} \omega^{e_{2}} m_{12} \omega^{c_{12}}=$ $p \omega^{e} m \omega^{c}+n_{2} m \omega^{c}=m p \omega^{e+c}=n_{1} \omega^{d_{1}}$ since $e>0$. Last we have $p_{1} \omega^{e_{1}} m_{21} \omega^{c_{21}}+$ $p_{2} \omega^{e_{2}} m_{22} \omega^{c_{22}}=0+n_{2} \omega^{d_{2}}$.

- Last, assume that $c>d_{2}$ and $e=0$, so that $c=d_{1}$. We take $p_{1} \omega^{e_{1}}=1$, $p_{2} \omega^{e_{2}}=p-1$. We set $m_{11} \omega^{c_{11}}=m \omega^{c}, m_{12} \omega^{c_{12}}=m \omega^{c}, m_{21} \omega^{c_{21}}=n_{2} \omega^{d_{2}}$ 
and $m_{22} \omega^{c_{22}}=0$. We have $m_{11} \omega^{c_{11}}+m_{21} \omega^{c_{21}}=m \omega^{c}+n_{2} \omega^{d_{2}}=m \omega^{c}$ since $c>d_{2}$ and $m_{12} \omega^{c_{12}}+m_{22} \omega^{c_{22}}=m \omega^{c}$. Next, we have $p_{1} \omega^{e_{1}} m_{11} \omega^{c_{11}}+$ $p_{2} \omega^{e_{2}} m_{12} \omega^{c_{12}}=m \omega^{c}+(p-1) m \omega^{c}=n_{1} \omega^{d_{1}}$ and last $p_{1} \omega^{e_{1}} m_{21} \omega^{c_{21}}+$ $p_{2} \omega^{e_{2}} m_{22} \omega^{c_{22}}=n_{2} \omega^{d_{2}}$.

Proof of Lemma 3. First, let $(\mu, \pi) \in !_{\mathbb{M}}(S \cdot R)$. Let $\varphi$ be a witness of $(\mu, \pi)$ for $S \cdot R$. For each $(a, c) \in S \cdot R$, let us choose $f(a, c) \in Y$ such that $(a, f(a, c)) \in R$ and $(f(a, c), c) \in S$. Let $\nu \in \mathbb{M}\langle Y\rangle$ be given by

$$
\nu(b)=\sum_{f(a, c)=b} \varphi(a, c) .
$$

This sum is finite, because $\varphi$ has finite support. Moreover, if $b \in \operatorname{supp}(\nu)$ then we must have $b=f(a, c)$ for some $(a, c) \in \operatorname{supp}(\varphi)$ and there are only finitely many such pairs $(a, c)$, so $\nu$ has finite support: $\nu \in !_{\mathbb{M}} Y$. We check that $(\mu, \nu) \in !_{\mathbb{M}} R$, and for this we exhibit a witness, namely $\sigma \in \mathbb{M}\langle X \times Y\rangle$ given by

$$
\sigma(a, b)=\sum_{f(a, c)=b} \varphi(a, c)
$$

Indeed, we have

$$
\begin{array}{ll}
\forall a \in X & \sum_{b \in Y} \sigma(a, b)=\sum_{b \in Y} \sum_{f(a, c)=b} \varphi(a, c)=\sum_{(a, c) \in R} \varphi(a, c)=\mu(a) \\
\forall b \in Y & \sum_{a \in X} \sigma(a, b)=\sum_{a \in X} \sum_{f(a, c)=b} \varphi(a, c)=\sum_{f(a, c)=b} \varphi(a, c)=\nu(b)
\end{array}
$$

One checks similarly that $(\nu, \pi) \in !_{\mathbb{M}} S$, and hence $(\mu, \pi) \in !_{\mathbb{M}} S \cdot !_{\mathbb{M}} R$.

Conversely, let $(\mu, \pi) \in !_{\mathbb{M}} S \cdot !_{\mathbb{M}} R$. Let $\nu \in !_{\mathbb{M}} Y$ be such that $(\mu, \nu) \in !_{\mathbb{M}} R$ and $(\nu, \pi) \in !_{\mathbb{M}} S$ and let $\sigma \in \mathbb{M}\langle X \times Y\rangle$ and $\tau \in \mathbb{M}\langle Y \times Z\rangle$ be corresponding witnesses. Let $b \in Y$. We have

$$
\sum_{a \in X} \sigma(a, b)=\sum_{c \in Z} \tau(b, c)=\nu(b) .
$$

By Lemma 1, we can find $\varphi^{b} \in \mathbb{M}\langle X \times Z\rangle$ such that

$$
\forall a \in X \quad \sigma(a, b)=\sum_{c \in Z} \varphi^{b}(a, c) \quad \text { and } \quad \forall a \in X \quad \tau(b, c)=\sum_{a \in X} \varphi^{b}(a, c) .
$$

Let $\varphi=\sum_{b \in \operatorname{supp}(\nu)} \varphi^{b}$. Let $a \in X$, we have

$$
\mu(a)=\sum_{b \in Y} \sigma(a, b)=\sum_{b \in Y} \sum_{c \in Z} \varphi^{b}(a, c)=\sum_{c \in Z} \sum_{b \in Y} \varphi^{b}(a, c)=\sum_{c \in Z} \varphi(a, c) .
$$

Similarly one show that $\pi(c)=\sum_{a \in X} \varphi(a, c)$. Last observe that if $(a, c) \in$ $\operatorname{supp}(\varphi)$, one has $(a, c) \in \operatorname{supp}\left(\varphi^{b}\right)$ for some $b$. For such a $b$ we have $(a, b) \in$ $\operatorname{supp}(\sigma) \subseteq R$ and $(b, c) \in \operatorname{supp}(\tau) \subseteq S$. This shows that $\operatorname{supp}(\varphi) \subseteq S \cdot R$, so that $\varphi$ is a witness of $(\mu, \pi)$ for $S \cdot R$, and hence $(\mu, \pi) \in !_{\mathbb{M} S} \cdot R$. 
Proof of Lemma 5. Let $R \in \operatorname{Rel}(X, Y)$. We must show that $R \cdot \mathrm{d}_{X}=\mathrm{d}_{Y} \cdot !_{\mathbb{M}} R$. Let $\mu \in !_{\mathbb{M}} X$ and $b \in Y$. Assume first that $(\mu, b) \in R \cdot \mathrm{d}_{X}$; this means that there exists $a \in X$ such that $(\mu, a) \in \mathrm{d}_{X}$ and $(a, b) \in R$. Hence we have $\mu=[a]$. We have $([a],[b]) \in !_{\mathbb{M}} R$ and hence also $(\mu, b) \in \mathrm{d}_{Y} \cdot !_{\mathbb{M}} R$.

Conversely assume that $(\mu, b) \in \mathrm{d}_{Y} \cdot !_{\mathbb{M}} R$, so that $(\mu,[b]) \in !_{\mathbb{M}} R$, and let $\sigma \in \mathbb{M}\langle X \times Y\rangle$ be a witness. We have $\sum_{a \in X} \sigma\left(a, b^{\prime}\right)=[b]\left(b^{\prime}\right)$ for each $b^{\prime} \in Y$. By Conditions (MS1) and (MS2), one has $\forall a \in X \sigma\left(a, b^{\prime}\right)=0$ for each $b^{\prime} \neq b$, and there exists $a \in X$ such that $\sigma(a, b)=1$ and $\sigma\left(a^{\prime}, b\right)=0$ for all $a^{\prime} \neq a$. We have therefore $\mu=[a]$. Since $(a, b) \in R$, this shows that $(\mu, b) \in R \cdot \mathrm{d}_{X}$ because $([a], a) \in \mathrm{d}_{X}$.

Proof of Lemma 6. Let $\sigma \in \mathbb{M}\langle X \times Y\rangle$ be a witness of $(\mu, \nu)$ for $R$. Let $b \in Y$. We have $\nu_{1}(b)+\nu_{2}(b)=\sum_{a \in X} \sigma(a, b)$. By Lemma 1 we can find $\varphi_{i}^{b} \in \mathbb{M}\langle X\rangle$ (for $i=1,2)$ such that $\nu_{i}(b)=\sum_{a \in X} \varphi_{i}^{b}(a)$ (for $\left.i=1,2\right)$ and $\sigma(a, b)=\varphi_{1}^{b}(a)+\varphi_{2}^{b}(a)$. Let $\sigma_{i}(a, b)=\varphi_{i}^{b}(a)$. Then $\sigma_{1}(a, b)+\sigma_{2}(a, b)=\sigma(a, b)$ and this shows that $\operatorname{supp}\left(\sigma_{i}\right) \subseteq R$ for $i=1,2$ (using Condition (MS1)). We have $\sum_{a \in X} \sigma_{i}(a, b)=$ $\nu_{i}(b)$ for each $i \in\{1,2\}$ and $b \in Y$. We set $\mu_{i}(a)=\sum_{b \in Y} \sigma_{i}(a, b)$. Then $\mu_{i} \in !_{\mathbb{M}} X$ for $i=1,2$ since $\sigma_{i}$ has finite support. Moreover $\left(\mu_{i}, \nu_{i}\right) \in !_{\mathbb{M}} R$ with witness $\sigma_{i}$ for $i=1,2$. We conclude because $\mu_{1}(a)+\mu_{2}(a)=\sum_{b \in Y}\left(\sigma_{1}(a, b)+\sigma_{2}(a, b)\right)=$ $\sum_{b \in Y} \sigma(a, b)=\mu(a)$.

Proof of Lemma 8. Let $X$ and $Y$ be sets and let $R \subseteq X \times Y$. Let $(\mu, \Pi) \in$ $!_{\mathbb{M}} X \times !_{\mathbb{M} !} !_{\mathbb{M}} Y$.

Assume first that $(\mu, \Pi) \in !_{\mathbb{M}} !_{\mathbb{M}} R \cdot \mathrm{p}_{X}$. Let $M \in !_{\mathbb{M} !} !_{\mathbb{M}} X$ be such that $(M, \Pi) \in$ $!_{\mathbb{M} !} !_{\mathbb{M}} R$ and $(\mu, M) \in \mathrm{p}_{X}$, that is $\Sigma(M)=\mu$. Let $\Theta \in \mathbb{M}\left\langle!_{\mathbb{M}} X \times !_{\mathbb{M}} Y\right\rangle$ be a witness of $(M, \Pi)$ for $!_{\mathbb{M}} R$. This means that

$$
\begin{aligned}
& \forall \mu^{\prime} \in !_{\mathbb{M} X} X\left(\mu^{\prime}\right)=\sum_{\pi^{\prime} \in !_{\mathbb{M} Y}} \Theta\left(\mu^{\prime}, \pi^{\prime}\right) \\
& \left.\forall \pi^{\prime} \in !_{\mathbb{M}} Y \quad \Pi\left(\pi^{\prime}\right)=\sum_{\mu^{\prime} \in !_{\mathbb{M}} X} \Theta\left(\mu^{\prime}, \pi^{\prime}\right)\right)
\end{aligned}
$$

Since $\operatorname{supp}(\Theta) \subseteq !_{\mathbb{M}} R$, by Lemma 4 , we have

$$
\left(\sum_{\mu^{\prime} \in !_{\mathbb{M}} X, \pi^{\prime} \in !_{\mathbb{M}} Y} \Theta\left(\mu^{\prime}, \pi^{\prime}\right) \mu^{\prime}, \sum_{\mu^{\prime} \in !_{\mathbb{M}} X, \pi^{\prime} \in !_{\mathbb{M}} Y} \Theta\left(\mu^{\prime}, \pi^{\prime}\right) \pi^{\prime}\right) \in !_{\mathbb{M}} R,
$$

that is $(\Sigma(M), \Sigma(\Pi)) \in !_{\mathbb{M}} R$. Therefore $(\mu, \Pi) \in \mathrm{p}_{Y} \cdot !_{\mathbb{M}} R$, since $(\Sigma(\Pi), \Pi) \in$ $\mathrm{p}_{Y}$.

Conversely, assume that $(\mu, \Pi) \in \mathrm{p}_{Y} \cdot !_{\mathbb{M}} R$, that is $(\mu, \Sigma(\Pi)) \in !_{\mathbb{M}} R$, that is $\left(\mu, \sum_{\pi \in !_{\mathbb{M}} Y} \Pi(\pi) \pi\right) \in !_{\mathbb{M}} R$. Let $R_{0} \subseteq R$ be finite and such that

$$
\left(\mu, \sum_{\pi \in !_{\mathbb{M}} Y} \Pi(\pi) \pi\right) \in !_{\mathbb{M}} R_{0} .
$$


Such an $R_{0}$ exists because $\mu$ and $\Pi$ have finite support. By Lemma 6 , one can find a family $\left(\mu^{\pi}\right)_{\pi \in \operatorname{supp}(\Pi)}$ of elements of $!_{\mathbb{M}} X$ such that $\mu=\sum_{\pi \in \operatorname{supp}(\Pi)} \mu^{\pi}$ and $\forall \pi \in \operatorname{supp}(\Pi),\left(\mu^{\pi}, \Pi(\pi) \pi\right) \in !_{\mathbb{M}} R_{0}$. Applying Lemma 7 , for each $\pi \in \operatorname{supp}(\Pi)$, we can find a family $\left(\mu_{i}^{\pi}\right)_{i=1}^{q\left(R_{0}\right)}$ of elements of $!_{\mathbb{M}} X$ and a family $\left(p_{i}^{\pi}\right)_{i=1}^{q\left(R_{0}\right)}$ of elements of $\mathbb{M}$ such that

- $\sum_{i=1}^{q\left(R_{0}\right)} p_{i}^{\pi}=\Pi(\pi)$

- $\sum_{i=1}^{q\left(R_{0}\right)} p_{i} \underline{\mu_{i}^{\pi}=\mu^{\pi}}$

- and $\forall i \in \overline{q\left(R_{0}\right)}\left(\mu_{i}^{\pi}, \pi\right) \in !_{\mathbb{M}} R$.

We define $M \in \mathbb{M}^{!} !_{\mathbb{M}} X$ by setting

$$
M=\sum_{\substack{\pi \in \operatorname{supp}(\Pi) \\ i \in \overline{q\left(R_{0}\right)}}} p_{i}^{\pi}\left[\mu_{i}^{\pi}\right] .
$$

This sum is finite because $\operatorname{supp}(\Pi)$ is a finite set.

We have

$$
\begin{aligned}
\Sigma(M) & =\sum_{\substack{\pi \in \operatorname{supp}(\Pi) \\
i \in q\left(R_{0}\right)}} p_{i}^{\pi} \mu_{i}^{\pi} \\
& =\sum_{\pi \in \operatorname{supp}(\Pi)} \sum_{i=1}^{q\left(R_{0}\right)} p_{i}^{\pi} \mu_{i}^{\pi}=\sum_{\pi \in \operatorname{supp}(\Pi)} \mu^{\pi}=\mu,
\end{aligned}
$$

so that $(\mu, M) \in \mathrm{p}_{X}$. Moreover we have $\forall \pi \in \operatorname{supp}(\Pi) \forall i \in \overline{q\left(R_{0}\right)}\left(\mu_{i}^{\pi}, \pi\right) \in !_{\mathbb{M}} R$ and hence by Lemma 4 we have

$$
\left(M, \sum_{\substack{\pi \in \operatorname{supp}(\Pi) \\ i \in \overline{q\left(R_{0}\right)}}} p_{i}^{\pi}[\pi]\right) \in !_{\mathbb{M} !} !_{\mathbb{M}} R
$$

and hence $(M, \Pi) \in !_{\mathbb{M} !} !_{\mathbb{M}} R$ because

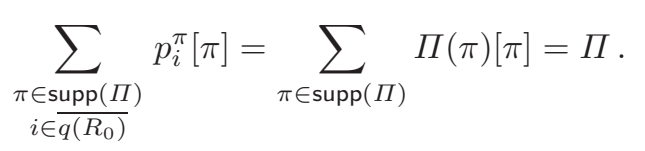

This shows that $(\mu, \Pi) \in !_{\mathbb{M}} !_{\mathbb{M}} R \cdot \mathrm{p}_{X}$ as announced.

Proof of Lemma 10. We prove that $\mathrm{p}_{!_{\mathbb{M}} X} \cdot \mathrm{p}_{X}=!_{\mathbb{M}} \mathrm{p}_{X} \cdot \mathrm{p}_{X}$, so let $(\mu, \mathcal{M}) \in$ $!_{\mathbb{M}} X \times !_{\mathbb{M}} !_{\mathbb{M}} !_{\mathbb{M}} X$. Assume first that $(\mu, \mathcal{M}) \in \mathrm{p}_{!_{\mathbb{M}} X} \cdot \mathrm{p}_{X}$, that is $\Sigma(\Sigma(\mathcal{M}))=\mu$. We define $M \in \mathbb{M}^{!} !_{\mathbb{M}}^{X}$ as follows:

$$
M(\nu)=\sum_{\substack{N \in !_{\mathbb{M}} !_{\mathbb{M}} X \\ \Sigma(N)=\nu}} \mathcal{M}(N) .
$$


Then $M \in !_{\mathbb{M} !} !_{\mathbb{M}} X$. Indeed, for each $\nu \in \operatorname{supp}(M)$ we can find $N \in \operatorname{supp}(\mathcal{M})$ such that $\nu \in \operatorname{supp}(N)$, hence $\operatorname{supp}(M) \subseteq \bigcup_{N \in \operatorname{supp}(\mathcal{M})} \operatorname{supp}(N)$ and this latter set is finite. We have

$$
\begin{aligned}
\Sigma(M) & =\sum_{\nu \in !_{\mathbb{M}} X} M(\nu) \nu \\
& =\sum_{\nu \in !_{\mathbb{M} X}}\left(\sum_{\Sigma(N)=\nu} \mathcal{M}(N)\right) \nu \\
& =\sum_{N \in !_{\mathbb{M} !} X} \mathcal{M}(N) \Sigma(N) \\
& =\Sigma(\Sigma(\mathcal{M}))=\mu
\end{aligned}
$$

and hence $(\mu, \Sigma(M)) \in \mathrm{p}_{X}$. Let $\Theta \in \mathbb{M}\left\langle!_{\mathbb{M}} X \times !_{\mathbb{M}} !_{\mathbb{M}} X\right\rangle$ be defined by

$$
\Theta(\nu, N)= \begin{cases}\mathcal{M}(N) & \text { if } \Sigma(N)=\nu \\ 0 & \text { otherwise }\end{cases}
$$

Then clearly $\operatorname{supp}(\Theta) \subseteq \mathrm{p}_{X}$. Moreover, we have $\sum_{\nu \in \ln _{X}} \Theta(\nu, N)=\mathcal{M}(N)$ for all $N \in !_{\mathbb{M} !} !_{\mathbb{M}} X$ and $\sum_{N \in !_{\mathbb{M} !} X} \Theta(\nu, N)=\sum_{\Sigma(N)=\nu} \mathcal{M}(N)=M(\nu)$ for all $\nu \in !_{\mathbb{M}} X$, by definition of $M$. This shows that $\Theta$ is a witness of $(M, \mathcal{M})$ for $\mathrm{p}_{X}$. So we have shown that $(M, \mathcal{M}) \in !_{\mathbb{M}} \mathrm{p}_{X}$ and therefore $(\mu, \mathcal{M}) \in !_{\mathbb{M}} \mathrm{p}_{X} \cdot \mathrm{p}_{X}$.

Assume conversely that $(\mu, \mathcal{M}) \in !_{\mathbb{M}} \mathrm{p}_{X} \cdot \mathrm{p}_{X}$. So let $M \in !_{\mathbb{M}} !_{\mathbb{M}} X$ be such that $(\mu, M) \in \mathrm{p}_{X}$ and $(M, \mathcal{M}) \in !_{\mathbb{M}} \mathrm{p}_{X}$. Let $\Theta \in \mathbb{M}\left\langle!_{\mathbb{M}} X \times !_{\mathbb{M}} !_{\mathbb{M}} X\right\rangle$ be a witness of $(M, \mathcal{M})$ for $\mathrm{p}_{X}$. Since $\operatorname{supp}(\Theta) \subseteq \mathrm{p}_{X}$, there is a map $H: !_{\mathbb{M}} !_{\mathbb{M}} X \rightarrow \mathbb{M}$ such that

$$
\Theta(\nu, N)= \begin{cases}H(N) & \text { if } \Sigma(N)=\nu \\ 0 & \text { otherwise. }\end{cases}
$$

For any $N \in !_{\mathbb{M} !} !_{\mathbb{M}} X$ we must have $\left.\mathcal{M}(N)=\sum_{\nu \in !_{\mathbb{M}} X} \Theta(\nu, N)\right)=H(N)$ so that $H=\mathcal{M}$. Therefore we have $M(\nu)=\sum_{\Sigma(N)=\nu} \mathcal{M}(N)$ for all $\nu \in !_{\mathbb{M}} X$. By Lemma 9 we have

$$
\begin{aligned}
\Sigma(\Sigma(\mathcal{M})) & =\sum_{N \in !_{\mathbb{M} !} X} \mathcal{M}(N) \Sigma(N) \\
& =\sum_{\nu \in !_{\mathbb{M} X}}\left(\sum_{\Sigma(N)=\nu} \mathcal{M}(N) \nu\right) \\
& =\sum_{\nu \in !_{\mathbb{M} X}} M(\nu) \nu \text { since } M(\nu)=\sum_{\Sigma(N)=\nu} \mathcal{M}(N) \\
& =\Sigma(M)=\mu
\end{aligned}
$$

Therefore $(\mu, \mathcal{M}) \in \mathrm{p}_{!_{\mathbb{M}} X} \cdot \mathrm{p}_{X}$. 
Proof of Proposition 5. The second bijection is $\mathrm{n}=\{(*,[])\}$. The first one is

$$
\mathrm{n}_{X_{1}, X_{2}}=\left\{\left(\left(\mu_{1}, \mu_{2}\right), \operatorname{in}_{1}\left(\mu_{1}\right)+\operatorname{in}_{2}\left(\mu_{2}\right) \mid \mu_{1} \in !_{\mathbb{M}} X_{1} \text { and } \mu_{2} \in !_{\mathbb{M}} X_{2}\right\}\right.
$$

where $\operatorname{in}_{i}(\mu)=\sum_{a \in X_{i}} \mu(a)[(i, a)]$. Let us check that this isomorphism is natural, so let $R_{i} \subseteq X_{i} \times Y_{i}$ for $i=1,2$. We must check that $\mathrm{n}_{Y_{1}, Y_{2}} \cdot\left(!_{\mathbb{M}} R_{1} \otimes !_{\mathbb{M}} R_{2}\right)=$ $!_{\mathbb{M}}\left(R_{1} \& R_{2}\right) \cdot \mathrm{n}_{X_{1}, X_{2}}$. So let $\mu_{i} \in !_{\mathbb{M}} X_{i}$ and $\nu_{i} \in !_{\mathbb{M}} Y_{i}$ for $i=1,2$.

Assume first that $\left(\left(\mu_{1}, \mu_{2}\right), \mathrm{in}_{1}\left(\nu_{1}\right)+\mathrm{in}_{2}\left(\nu_{2}\right)\right) \in \mathrm{n}_{Y_{1}, Y_{2}} \cdot\left(!_{\mathbb{M}} R_{1} \otimes !_{\mathbb{M}} R_{2}\right)$. This means that one can find $\nu_{i}^{\prime} \in !_{\mathbb{M}} Y_{i}$ for $i=1,2$ such that $\left(\mu_{i}, \nu_{i}^{\prime}\right) \in !_{\mathbb{M}} R_{i}$ for $i=1,2$, and $\left(\left(\nu_{1}^{\prime}, \nu_{2}^{\prime}\right), \mathrm{in}_{1}\left(\nu_{1}\right)+\mathrm{in}_{2}\left(\nu_{2}\right)\right) \in \mathrm{n}_{Y_{1}, Y_{2}}$. This means that $\nu_{i}=\nu_{i}^{\prime}$ for $i=1,2$. Since $\left(\mu_{1}, \nu_{1}\right) \in !_{\mathbb{M}} R_{1}$, we have $\left(\operatorname{in}_{1}\left(\mu_{1}\right), \operatorname{in}_{1}\left(\nu_{1}\right)\right) \in !_{\mathbb{M}}\left(R_{1} \& R_{2}\right)$ and similarly $\left(\operatorname{in}_{2}\left(\mu_{2}\right), \operatorname{in}_{2}\left(\nu_{2}\right)\right) \in !_{\mathbb{M}}\left(R_{1} \& R_{2}\right)$ and hence $\left(\operatorname{in}_{1}\left(\mu_{1}\right)+\operatorname{in}_{2}\left(\mu_{2}\right), \operatorname{in}_{1}\left(\nu_{1}\right)+\right.$ $\left.\operatorname{in}_{2}\left(\nu_{2}\right)\right) \in !_{\mathbb{M}}\left(R_{1} \& R_{2}\right)$ by Lemma 4 . But $\left(\left(\mu_{1}, \mu_{2}\right), \operatorname{in}_{1}\left(\mu_{1}\right)+\operatorname{in}_{2}\left(\mu_{2}\right)\right) \in \mathrm{n}_{X_{1}, X_{2}}$ and we have therefore $\left(\left(\mu_{1}, \mu_{2}\right), \mathrm{in}_{1}\left(\nu_{1}\right)+\mathrm{in}_{2}\left(\nu_{2}\right)\right) \in !_{\mathbb{M}}\left(R_{1} \& R_{2}\right) \cdot \mathrm{n}_{X_{1}, X_{2}}$.

Assume conversely that $\left(\left(\mu_{1}, \mu_{2}\right), \mathrm{in}_{1}\left(\nu_{1}\right)+\mathrm{in}_{2}\left(\nu_{2}\right)\right) \in !_{\mathbb{M}}\left(R_{1} \& R_{2}\right) \cdot \mathrm{n}_{X_{1}, X_{2}}$, so that there exist $\mu_{i}^{\prime} \in !_{\mathbb{M}} X_{i}$ for $i=1,2$ with $\left(\left(\mu_{1}, \mu_{2}\right), \operatorname{in}_{1}\left(\mu_{1}^{\prime}\right)+\operatorname{in}_{2}\left(\mu_{2}^{\prime}\right)\right) \in \mathrm{n}_{X_{1}, X_{2}}$ and $\left(\mathrm{in}_{1}\left(\mu_{1}^{\prime}\right)+\mathrm{in}_{2}\left(\mu_{2}^{\prime}\right), \mathrm{in}_{1}\left(\nu_{1}\right)+\mathrm{in}_{2}\left(\nu_{2}\right)\right) \in !_{\mathbb{M}}\left(R_{1} \& R_{2}\right)$. Therefore $\mu_{i}^{\prime}=\mu_{i}$ for $i=1,2$ and hence $\left(\operatorname{in}_{1}\left(\mu_{1}\right)+\operatorname{in}_{2}\left(\mu_{2}\right), \operatorname{in}_{1}\left(\nu_{1}\right)+\operatorname{in}_{2}\left(\nu_{2}\right)\right) \in !_{\mathbb{M}}\left(R_{1} \& R_{2}\right)$. Let $\varphi$ be a witness of $\left(\mathrm{in}_{1}\left(\mu_{1}\right)+\mathrm{in}_{2}\left(\mu_{2}\right), \mathrm{in}_{1}\left(\nu_{1}\right)+\mathrm{in}_{2}\left(\nu_{2}\right)\right)$ for $R_{1} \& R_{2}$. Since $\operatorname{supp}(\varphi) \subseteq R_{1} \& R_{2}$, we have $\left(\mu_{i}, \nu_{i}\right) \in !_{\mathbb{M}} R_{i}$ for $i=1,2$ : take $\varphi_{i} \in \mathbb{M}\left\langle X_{i} \times Y_{i}\right\rangle$ defined by $\varphi_{i}\left(a_{i}, b_{i}\right)=\varphi\left(\left(i, a_{i}\right),\left(i, b_{i}\right)\right)$, then $\varphi_{i}$ is a witness of $\left(\mu_{i}, \nu_{i}\right)$ for $R_{i}$. It follows that $\left(\left(\mu_{1}, \mu_{2}\right),\left(\nu_{1}, \nu_{2}\right)\right) \in !_{\mathbb{M}} R_{1} \otimes !_{\mathbb{M}} R_{2}$ and therefore $\left(\left(\mu_{1}, \mu_{2}\right), \mathrm{in}_{1}\left(\nu_{1}\right)+\right.$ $\left.\operatorname{in}_{2}\left(\nu_{2}\right)\right) \in \mathrm{n}_{Y_{1}, Y_{2}} \cdot\left(!_{\mathbb{M}} R_{1} \otimes !_{\mathbb{M}} R_{2}\right)$. 